\title{
Random Forests Approach for Causal Inference with Clustered Observational Data
}

\author{
Youmi Suk ${ }^{* 1}$, Hyunseung Kang ${ }^{\dagger 2}$, and Jee-Seon Kim ${ }^{\ddagger 1}$ \\ ${ }^{1}$ Department of Educational Psychology, University of Wisconsin-Madison \\ ${ }^{2}$ Department of Statistics, University of Wisconsin-Madison
}

July 23, 2020

\begin{abstract}
There is a growing interest in using machine learning (ML) methods for causal inference due to their (nearly) automatic and flexible ability to model key quantities such as the propensity score or the outcome model. Unfortunately, most ML methods for causal inference have been studied under single-level settings where all individuals are independent of each other and there is little work in using these methods with clustered or nested data, a common setting in education studies. This paper investigates using one particular ML method based on random forests known as Causal Forests to estimate treatment effects in multilevel observational data. We conduct simulation studies under different types of multilevel data, including two-level, three-level, and cross-classified data. Our simulation study shows that when the ML method is supplemented with estimated propensity scores from multilevel models that account for clustered/hierarchical structure, the modified ML method outperforms pre-existing methods in a wide variety of settings. We conclude by estimating the effect of private math lessons in the Trends in International Mathematics and Science Study data, a large-scale educational assessment where students are nested within schools.
\end{abstract}

Keywords: Causal inference, Machine learning methods, Multilevel propensity score matching, Multilevel observational data, Hierarchical linear modeling

\section{Introduction}

In the social sciences, when studying treatment effects with observational data, study units are naturally clustered together. For example, in the Trends in International Mathematics and Science Study (TIMSS) and the Programme for International Student Assessment (PISA), students, the study unit, are clustered/nested at the school level, and schools are clustered/nested at the country level; this type of data is known as three-level data or, more broadly, multilevel/hierarchical data. In cross-classified data, students belong to two clusters simultaneously, say student's cluster membership is defined by school and neighborhood (Raudenbush \& Bryk, 2002). The goal of this paper is to estimate the average treatment effect (ATE) in multilevel observational data where the treatment is assigned at the unit level (e.g., students instead of schools or neighborhoods).

Traditionally, the most popular way to estimate the ATE in clustered observational data is by multilevel propensity score methods (Hong \& Raudenbush, 2006; Steiner, Kim, \& Thoemmes, 2012; Kim \& Seltzer, 2007; Thoemmes \& West, 2011). Briefly, a propensity score is a study unit's conditional probability of receiving treatment given observed pre-treatment covariates (Rosenbaum \& Rubin, 1983) and

\footnotetext{
*ysuk@wisc.edu

${ }^{\dagger}$ hyunseung@stat.wisc.edu

$\ddagger$ jeeseonkim@wisc.edu

This article has been accepted for publication in Multivariate Behavioral Research, published by Taylor \& Francis.
} 
each study unit has a propensity score ranging from 0 to 1 . Propensity score methods utilize propensity scores to balance covariates between treated and control groups by using matching, stratification, or weighting. Multilevel propensity score methods use propensity scores, but also account for the underlying clustered/hierarchical structure in multilevel data, typically by fitting a multilevel propensity score model; see Section 10 of Leite (2016) for details. If selection is strongly ignorable, i.e., the treatment assignment is as-if random conditional on observed pre-treatment covariates, and the propensity score model is correctly specified, these aforementioned methods can consistently estimate the ATE. Also, propensity score methods can be combined with models for the outcome to form doubly robust estimators; these estimators are consistent for the ATE as long as either the propensity score model or the outcome model is correctly specified.

Recently, using machine learning (ML) algorithms has become a popular way to estimate the ATE (McCaffrey, Ridgeway, \& Morral, 2004; van der Laan, Polley, \& Hubbard, 2007; van der Laan \& Rose, 2011; Westreich, Lessler, \& Funk, 2010; Keller, Kim, \& Steiner, 2015; Athey \& Imbens, 2016; Hill, 2011; Chernozhukov et al., 2018). An attractive feature of ML-based methods is that they flexibly and, in some cases, automatically estimate the propensity score model or the outcome regression model. However, many of these works focus on what we call single-level data settings where all study units are (i) independent of each other and (ii) come from the same population; in short, the study units are assumed to be independent and identically distributed (i.i.d.). If the i.i.d. assumption is satisfied, many ML-based methods are consistent for the ATE. In contrast, if study units are clustered or nested, such as the students in the TIMSS data, the i.i.d. assumption no longer holds and there is no guarantee that these ML-based methods produce a consistent estimate for the ATE. Indeed, as highlighted by Carvalho, Feller, Murray, Woody, and Yeager (2019), existing ML methods should be modified, say by using different tuning parameters or re-designing sampling splits, to respect the underlying clustering or hierarchical structure and to produce a more precise and consistent estimate of the treatment effect in multilevel data. However, it remains an open question as to how to exactly make such modifications for different kinds of ML methods.

The goal of this paper is to study how to modify ML-based methods in order to estimate the ATE in multilevel observational data. We focus on one ML method based on random forests called Causal Forests (Wager \& Athey, 2018; Athey, Tibshirani, \& Wager, 2019) which is a popular ML-based method in causal inference. We consider three types of multilevel data: two-level, three-level, and cross-classified data. These settings, in general, violate the underlying assumptions that validate Causal Forests as a consistent and asymptotically Normal estimator of the ATE because of dependencies between subjects. We study three simple ways to modify Causal Forests in order to account for the underlying hierarchical structure. The first modification injects Causal Forests with propensity scores from a multilevel logistic regression. The second modification uses cluster labels that denote each level of the hierarchy and changes how sample splitting is done inside Causal Forests. The third modification combines the two modifications. We compare how well these modified ML methods perform compared to ML methods without any modifications, traditional multilevel propensity score methods, and doubly robust methods where the latter two use parametric multilevel models by measuring the absolute relative bias, standard deviation, and mean-squared error. Lastly, we demonstrate our findings by studying the effect of private math lessons on students' math achievement scores from the 2015 Korea TIMSS data.

Overall, we found that in a wide range of scenarios, the modified Causal Forests using an estimated propensity score from a multilevel logistic regression was competitive to doubly robust estimators with correctly specified propensity score and outcome regression models. Also, the modified Causal Forests had smaller mean-squared error than traditional multilevel propensity score methods or the original Causal Forests without any modifications. This phenomenon generally held true even when the multilevel propensity score model in the modified Causal Forest was moderately mis-specified. More broadly, we believe that our modifications of Causal Forests based on the multilevel propensity score can serve as a template to modify other ML-based methods in causal inference when they are used in observational studies with hierarchical/clustered structures.

\section{Causal Inference with Clustered Observational Data}

We use the Neyman-Rubin potential outcomes notation (Neyman, 1923; Rubin, 1974) and its extension to multilevel/clustered settings by Hong and Raudenbush (2006) to formalize causal effects. Suppose that there are $N$ total individuals, indexed by $i j$ where $i$ indexes study units within a cluster and $j$ indexes clusters. Let $Z_{i j}$ denote the treatment assignment of individual $i$ in cluster $j$, with $Z_{i j}=1$ representing a treated individual and $Z_{i j}=0$ representing an untreated individual; as noted earlier, this paper focuses 
on studies where the treatment is assigned at the individual level, not at the cluster level. Let $Y_{i j}(1)$ be the potential outcome if individual $i$ in cluster $j$ were to be treated $\left(Z_{i j}=1\right)$ and let $Y_{i j}(0)$ be the potential outcome if individual $i$ within cluster $j$ were to be untreated $\left(Z_{i j}=0\right)$. The observed outcome is $Y_{i j}=Z_{i j} Y_{i j}(1)+\left(1-Z_{i j}\right) Y_{i j}(0)$. We remark that the notation assumes Stable Unit Treatment Value Assumption (SUTVA; Rubin, 1986); for more information on SUTVA in multilevel settings, see Hong and Raudenbush $(2006,2013)$. Finally, let $\boldsymbol{X}_{i j}$ and $\boldsymbol{W}_{j}$ denote individual-level and cluster-level pretreatment covariates, respectively. Individual-level covariates $\boldsymbol{X}_{i j}$ are covariates whose values vary across individuals, say gender, age, and socioeconomic status, and cluster-level covariates $\boldsymbol{W}_{j}$ are covariates whose values are the same among individuals in the same cluster, say school type and school climate. Typically, cluster-level covariates define the underlying clustering or hierarchical structure in multilevel data.

The target estimand of interest is the ATE and is defined as the average linear contrast between two potential outcomes, i.e., $\tau=E\left[Y_{i j}(1)-Y_{i j}(0)\right]$. In a completely randomized experiment, the ATE can be estimated by taking the difference between the outcomes of treated and untreated groups. However, in observational studies, this approach may lead to bias because the treated and untreated groups are no longer similar with respect to their observed and unobserved covariates.

The typical set of working assumptions for estimating the ATE in observational studies is as follows:

Assumption 1 (Unconfoundness): $Y_{i j}(1), Y_{i j}(0) \perp Z_{i j} \mid \boldsymbol{X}_{i j}, \boldsymbol{W}_{j}$

Assumption 2 (Positivity): $0<e\left(\boldsymbol{X}_{i j}, \boldsymbol{W}_{j}\right)=\operatorname{Pr}\left(Z_{i j}=1 \mid \boldsymbol{X}_{i j}, \boldsymbol{W}_{j}\right)<1$

Here, $\perp$ represents independence between two random variables. In a nutshell, Assumptions 1 and 2 state that conditional on the observed individual-level and cluster-level covariates $\boldsymbol{X}_{i j}$ and $\boldsymbol{W}_{j}$, the treatment is as-if randomly assigned to each individual with non-zero probability. Assumptions 1 and 2 combined are often referred to as strong ignorability (Rosenbaum \& Rubin, 1983). An important idea arising from Assumptions 1 and 2 is that the propensity score $e\left(\boldsymbol{X}_{i j}, \boldsymbol{W}_{j}\right)$ is the coarsest balancing score (Rosenbaum \& Rubin, 1983). Specifically, conditional on the propensity score, the distribution of pre-treatment covariates is independent of treatment assignment, i.e., $\boldsymbol{X}_{i j}, \boldsymbol{W}_{j} \perp Z_{i j} \mid e\left(\boldsymbol{X}_{i j}, \boldsymbol{W}_{j}\right)$, and the distribution of covariates is identical or "balanced" between the treated and untreated groups. This latter idea serves as the basis for propensity score methods where treated and untreated units are matched, weighed, or stratified based on their propensity scores. The next section reviews some of these propensity score methods for multilevel data.

\section{Methods of Estimating the Average Treatment Effect in Multi- level Data}

\subsection{Multilevel Propensity Score Methods via Weighting}

Among various propensity score methods in multilevel data, weighting methods are one of the most frequently in practice. Briefly, weighting methods use propensity scores as sampling weights to weigh the outcomes between treated and untreated groups in order to unbiasedly estimate the ATE (Schafer \& Kang, 2008; Stuart, 2010; Lunceford \& Davidian, 2004). The most well-known weighting method is the inverse-propensity weighting (IPW) estimator, which is the weighted difference in the outcomes $Y_{i j}$ of treated and untreated groups where the weights are the inverse of the estimated propensity scores:

$$
\begin{aligned}
\omega_{z, i j}^{\mathrm{IPW}} & =\frac{z}{e\left(\boldsymbol{X}_{i j}, \boldsymbol{W}_{j}\right)}+\frac{1-z}{1-e\left(\boldsymbol{X}_{i j}, \boldsymbol{W}_{j}\right)} \\
\hat{\tau}_{\mathrm{IPW}} & =\frac{1}{N} \sum_{i j} Y_{i j} Z_{i j} \omega_{1, i j}^{\mathrm{IPW}}-\frac{1}{N} \sum_{i j} Y_{i j}\left(1-Z_{i j}\right) \omega_{0, i j}^{\mathrm{IPW}}
\end{aligned}
$$

(Hong \& Hong, 2009) proposed another weighting estimator called the marginal mean weighting through stratification (MMW-S). MMW-S estimator, like the IPW estimator, takes the weighted difference in outcome between treated and untreated groups. But MMW-S stratifies the propensity score into percentiles and uses sample frequencies within each stratum as weights. A bit more formally, consider the observed frequencies $O_{z s}$ for each treatment status $z \in\{0,1\}$ in stratum $s \in\{1,2, \ldots, S\}$ defined by percentiles of $e\left(\boldsymbol{X}_{i j}, \boldsymbol{W}_{j}\right)$ on the logit scale (or probability scale). Define the expected frequencies $E_{z s}$ for each treatment status $z$ in stratum $s$ to be $E_{z s}=O_{(z \cdot)} \times O_{(\cdot s)} / O_{(\cdot)}$; the dots in the subscript (.) denote sums 
over $z$ or $s$. Then, MMW-S weights are computed by dividing the expected frequencies by the observed frequencies, $E_{z s} / O_{z s}$, and the MMW-S estimator of the ATE is:

$$
\begin{aligned}
& \omega_{z, i j}^{\mathrm{MMW}-\mathrm{S}}= \begin{cases}\frac{E_{z 1}}{O_{z 1}} & \text { if } e\left(\boldsymbol{X}_{i j}, \boldsymbol{W}_{j}\right) \text { in stratum } 1 \\
\vdots & \\
\frac{E_{z S}}{O_{z S}} & \text { if } e\left(\boldsymbol{X}_{i j}, \boldsymbol{W}_{j}\right) \text { in stratum } S\end{cases} \\
& \hat{\tau}_{\mathrm{MMW}-\mathrm{S}}=\frac{1}{N} \sum_{i j} Y_{i j} Z_{i j} \omega_{1, i j}^{\mathrm{MMW}-\mathrm{S}}-\frac{1}{N} \sum_{i j} Y_{i j}\left(1-Z_{i j}\right) \omega_{0, i j}^{\mathrm{MMW}-\mathrm{S}}
\end{aligned}
$$

The use of MMW-S requires researchers to choose the number of strata $S$. Hong (2010) suggested choosing $S$ to be the minimal number of strata that achieves within-stratum covariate balance in at least $95 \%$ of the observed covariates. We follow this advice in our simulation and real data studies and use covariates' standardized mean and variance within strata as our measure of within-stratum balance; see the Appendix C for details.

A key consideration in using weighting estimators in multilevel data is a model for the propensity score $e\left(\boldsymbol{X}_{i j}, \boldsymbol{W}_{j}\right)$. Broadly speaking, in two-level data, there are two main strategies for modeling the propensity score and estimating the ATE: a within-cluster strategy and an across-cluster strategy (Leite, 2016; Steiner et al., 2012). A within-cluster strategy estimates a propensity score model for each cluster with only individual-level covariates. Each propensity score model is trained using only study units within the cluster and is usually a logistic regression commonly found in single-level i.i.d. settings. Then, using one of the weighting estimators above, cluster-level treatment effects are computed and then aggregated to estimate the ATE. In contrast, an across-cluster strategy estimates a single joint propensity score model across all clusters. The joint propensity score model is typically a multilevel logistic regression with either random-effects or fixed-effects; random-effects models usually have both individual-level and cluster-level covariates, whereas fixed-effects models have individual-level covariates and cluster-level dummy variables (Leite, 2016; Steiner et al., 2012).

Generally speaking, weighting estimators based on a propensity score model from a within-cluster strategy is more robust to biases from unmeasured cluster-level covariates than those from an acrosscluster strategy; one can make a joint propensity score more robust to unobserved cluster-level covariates by adding interaction terms between cluster labels and covariates. However, when there are strong selection processes at work or cluster sizes are small, overlap is hard to achieve with propensity score estimates based on a within-cluster strategy, and in such settings, an across-cluster strategy is preferred (Kim \& Seltzer, 2007; Thoemmes \& West, 2011; Steiner et al., 2012). Given that the size of clusters in our real data is not large and a within-cluster strategy is often not feasible in these settings (Arpino \& Mealli, 2011; Steiner et al., 2012), this paper uses an across-cluster strategy where a single joint propensity score model is estimated using a multilevel logistic regression model with random effects.

Finally, we remark that there are other types of multilevel propensity score methods, such as two-stage matching (Rickles \& Seltzer, 2014), preferential matching (Arpino \& Cannas, 2016), and within-class matching (Kim \& Steiner, 2015), and most multilevel propensity score methods are tailored for two-level data.

\subsection{Doubly Robust Methods}

Doubly robust (DR) methods are estimators that provide consistent estimates of the ATE as long as the propensity score or the outcome model is correctly specified, but not necessarily both (Schafer \& Kang, 2008; Scharfstein, Rotnitzky, \& Robins, 1999). Here, we present a DR estimator based on fitting weighted outcome regression models for treated and untreated units, i.e., $E\left[Y_{i j} \mid \boldsymbol{X}_{i j}, \boldsymbol{W}_{j}, Z_{i j}=z\right]=$ $m_{z}\left(\boldsymbol{X}_{i j}, \boldsymbol{W}_{j}, \boldsymbol{\beta}_{z}\right)$ where $\boldsymbol{\beta}_{z}$ is the parameter of the outcome regression function $m_{z}$ for treatment group $z \in\{0,1\}$, where the weights in the regression are from the IPW estimator or MMW-S estimator (Schafer \& Kang, 2008; Leite, 2016). Specifically, the DR estimator based on weights from the IPW estimator is:

$$
\begin{aligned}
\hat{\boldsymbol{\beta}}_{z}^{\mathrm{IPW}} & =\operatorname{argmin}_{\boldsymbol{\beta}_{z}} \sum_{i j, Z_{i j}=z} \omega_{z, i j}^{\mathrm{IPW}}\left[Y_{i j}-m_{z}\left(\boldsymbol{X}_{i j}, \boldsymbol{W}_{j}, \boldsymbol{\beta}_{z}\right)\right]^{2} \\
\hat{\tau}_{\mathrm{DR} \mathrm{IPW}} & =\frac{1}{N} \sum_{i j} m_{1}\left(\boldsymbol{X}_{i j}, \boldsymbol{W}_{j}, \hat{\boldsymbol{\beta}}_{1}^{\mathrm{IPW}}\right)-\frac{1}{N} \sum_{i j} m_{0}\left(\boldsymbol{X}_{i j}, \boldsymbol{W}_{j}, \hat{\boldsymbol{\beta}}_{0}^{\mathrm{IPW}}\right)
\end{aligned}
$$


and the DR estimator based on weights from the MMW-S estimator is:

$$
\begin{aligned}
\hat{\boldsymbol{\beta}}_{z}^{\mathrm{MMW}-\mathrm{S}} & =\operatorname{argmin}_{\boldsymbol{\beta}_{z}} \sum_{i j, Z_{i j}=z} \omega_{z, i j}^{\mathrm{MMW}-\mathrm{S}}\left[Y_{i j}-m_{z}\left(\boldsymbol{X}_{i j}, \boldsymbol{W}_{j}, \boldsymbol{\beta}_{z}\right)\right]^{2} \\
\hat{\tau}_{\mathrm{DR} \text { MMW-S }} & =\frac{1}{N} \sum_{i j} m_{1}\left(\boldsymbol{X}_{i j}, \boldsymbol{W}_{j}, \hat{\boldsymbol{\beta}}_{1}^{\mathrm{MMW}-\mathrm{S}}\right)-\frac{1}{N} \sum_{i j} m_{0}\left(\boldsymbol{X}_{i j}, \boldsymbol{W}_{j}, \hat{\boldsymbol{\beta}}_{0}^{\mathrm{MMW}-\mathrm{S}}\right)
\end{aligned}
$$

In multilevel settings, typical models for $m_{z}$ are random-effects or fixed-effects linear regression models. In this paper, we will use random-effects linear regression models for $m_{z}$.

\subsection{Causal Forests and Modifications for Multilevel Data}

Recently, there is a growing trend in using flexible, non-parametric ML algorithms to estimate $e$ or $m_{z}$ without having to specify the functional form of these models. For example, targeted maximum likelihood estimators of the ATE (van der Laan \& Rose, 2011) often utilize an ensemble learner called SuperLearner (van der Laan et al., 2007), which combines different ML algorithms such as the Lasso, K-nearest matching, generalized additive models (GAMs), generalized linear models (GLMs), random forests, and multivariate adaptive regression splines (MARS), to flexibly estimate the propensity score and the outcome model. Causal Forests is another popular ML method for estimating the ATE as well as the conditional average treatment effect (CATE); we remark that averaging across unbiased estimates of the CATE leads to an unbiased estimator for the ATE. In this paper, we focus on Causal Forests and see how it performs in multilevel observational data.

We briefly sketch out the details of the Causal Forest algorithm; see Wager and Athey (2018), Athey et al. (2019), and Athey and Wager (2019) for details. Let $m(\boldsymbol{x}, \boldsymbol{w})=E\left[Y_{i j} \mid \boldsymbol{X}_{i j}=\boldsymbol{x}, \boldsymbol{W}_{j}=\boldsymbol{w}\right]$ be the conditional mean of the outcome given a specific value of covariates $\boldsymbol{x}, \boldsymbol{w}$. Let $\hat{m}^{-i j}(\boldsymbol{x}, \boldsymbol{w})$ be an estimate of this conditional mean at $\boldsymbol{x}$ and $\boldsymbol{w}$ where the estimate does not use study unit $i j$ 's data. Similarly, let $\hat{e}^{-i j}(\boldsymbol{x}, \boldsymbol{w})$ be an estimate of the propensity score where the estimate does not use study unit $i j$ 's data; these type of estimates are also known as out-of-bag leave-one-out estimates in machine learning. In the Causal Forests algorithm, the default estimates of $\hat{m}^{-i j}(\boldsymbol{x}, \boldsymbol{w})$ and $\hat{e}^{-i j}(\boldsymbol{x}, \boldsymbol{w})$ are computed by a honest random forest algorithm (see Procedure 1 in Wager and Athey (2018) for one example), although a consistent estimator with certain statistical properties will suffice; see Section 6.1.1 of (Athey et al., 2019) for the exact conditions. For example, if the propensity score is known as in a completely randomized experiment, we can plug in the probability of treatment from the experimental design as $\hat{e}^{-i j}(\boldsymbol{x}, \boldsymbol{w})$ and this satisfies the aforementioned statistical properties. A Causal Forest estimator of the CATE at covariates $\boldsymbol{x}, \boldsymbol{w}$, denoted as $\hat{\tau}(\boldsymbol{x}, \boldsymbol{w})$, is essentially a weighted linear regression of residualized outcome $\tilde{Y}_{i j}=Y_{i j}-\hat{m}^{-i j}(\boldsymbol{x}, \boldsymbol{w})$ and a single residualized regressor $\tilde{Z}_{i j}=Z_{i j}-\hat{e}^{-i j}(\boldsymbol{x}, \boldsymbol{w})$ and can be written as follows:

$$
\hat{\tau}(\boldsymbol{x}, \boldsymbol{w})=\frac{\sum_{i j} \alpha_{i j}(\boldsymbol{x}, \boldsymbol{w})\left(Y_{i j}-\hat{m}^{(-i j)}\left(\boldsymbol{X}_{i j}, \boldsymbol{W}_{j}\right)\right)\left(Z_{i j}-\hat{e}^{(-i j)}\left(\boldsymbol{X}_{i j}, \boldsymbol{W}_{j}\right)\right)}{\sum_{i j} \alpha_{i j}(\boldsymbol{x}, \boldsymbol{w})\left(Z_{i j}-\hat{e}^{(-i j)}\left(\boldsymbol{X}_{i j}, \boldsymbol{W}_{j}\right)\right)^{2}}
$$

Here, $0 \leq \alpha_{i j}(\boldsymbol{x}, \boldsymbol{w}) \leq 1$ weighs each data point ij's contribution to $\hat{\tau}(\boldsymbol{x}, \boldsymbol{w})$ based on how far $i j$ 's covariates, i.e., $\boldsymbol{X}_{i j}, \boldsymbol{W}_{j}$, are away from $\boldsymbol{x}, \boldsymbol{w}$. Roughly speaking, a high $\alpha_{i j}(\boldsymbol{x}, \boldsymbol{w})$ generally indicates that data point $i j$ contributes a large portion to $\hat{\tau}(\boldsymbol{x}, \boldsymbol{w})$, whereas a small $\alpha_{i j}(\boldsymbol{x}, \boldsymbol{w})$ generally indicates that data point $i j$ contributes a small portion to $\hat{\tau}(\boldsymbol{x}, \boldsymbol{w})$.

Computing the weights $\alpha_{i j}(\boldsymbol{x}, \boldsymbol{w})$ is based on a modified recursive partitioning algorithm with $\tilde{Y}_{i j}, \tilde{Z}_{i j}$, $\boldsymbol{X}_{i j}, \boldsymbol{W}_{j}$. Specifically, suppose we obtain $B$ bootstrap replicates and let $b=1, \ldots, B$ be one of those replicates. For each $b$ th replicate, take a random subsample without replacement of size $s$ from $n$ total samples in the $b$ th replicate. The algorithm randomly partitions the $s$ subsampled data into two equallysized data, say subsets $\mathcal{J}_{1}$ and $\mathcal{J}_{2}$. Using $\mathcal{J}_{1}$, the algorithm initializes a "parent" node $\mathcal{P}$, which represents all the data in $\mathcal{J}_{1}$, and computes pseudo-outcomes $\rho_{i j}$ for data in $\mathcal{P}$

$$
\rho_{i j}=D_{\mathcal{P}}^{-1}\left(\tilde{Z}_{i j}-\tilde{Z}_{\mathcal{P}}\right)\left(\tilde{Y}_{i j}-\tilde{Y}_{\mathcal{P}}-\left(\tilde{Z}_{i j}-\tilde{Z}_{\mathcal{P}}\right) \hat{\beta}_{\mathcal{P}}\right), \quad D_{\mathcal{P}}=\frac{1}{I(i j \in \mathcal{P})} \sum_{i j \in \mathcal{P}}\left(\tilde{Z}_{i j}-\tilde{Z}_{\mathcal{P}}\right)^{2}
$$

Here, $\tilde{Y}_{\mathcal{P}}$ and $\tilde{Z}_{\mathcal{P}}$ represent averages of $\tilde{Y}_{i j}$ and $\tilde{Z}_{i j}$, respectively, among data in the parent node $\mathcal{P}$ and $\hat{\beta}_{\mathcal{P}}$ represents a linear regression between $\tilde{Y}_{i j}$ and $\tilde{Z}_{i j}$ among data points in $\mathcal{P}$. It then uses standard 
Classification and Regression Trees (CART) (Breiman, Friedman, Olshen, \& Stone, 1984) with the psuedooutcomes $\rho_{i j}$ and covariates $\boldsymbol{X}_{i j}, \boldsymbol{W}_{j}$ to find a partition of the parent node $\mathcal{P}$ into two non-overlapping children nodes $\mathcal{C}_{1}$ and $\mathcal{C}_{2}$ such that the following argument is maximized

$$
\operatorname{argmax}_{\mathcal{C}_{1}, \mathcal{C}_{2}} \frac{\sum_{i j \in \mathcal{C}_{1}} \rho_{i j}^{2}}{\sum_{i j \in \mathcal{P}} I\left(i j \in \mathcal{C}_{1}\right)}+\frac{\sum_{i j \in \mathcal{C}_{2}} \rho_{i j}^{2}}{\sum_{i j \in \mathcal{P}} I\left(i j \in \mathcal{C}_{2}\right)}
$$

Roughly speaking, the argmax above finds partitions $\mathcal{C}_{1}$ and $\mathcal{C}_{2}$ of $\mathcal{P}$ such that the variance of the pseudooutcomes within each partition is maximized. For example, if one of the covariates is sex (male or female), $\mathcal{C}_{1}$ may be study units who are female and $\mathcal{C}_{2}$ may be study units who are male. It may choose these partition based on sex if it maximizes the equation above. Once it finds a partition $\mathcal{C}_{1}, \mathcal{C}_{2}$, it relabels $\mathcal{C}_{1}$ as the parent $\mathcal{P}$ and repeats the procedure; it also does the same for the other child node $\mathcal{C}_{2}$. The procedure stops after it reaches a stopping criterion and the final output is a partition of the covariate space that is represented as a binary tree. It then uses the other subset $\mathcal{J}_{2}$ to count the proportion of data in $\mathcal{J}_{2}$ that fall inside each of the terminal leaf nodes in the binary tree. This process is repeated across $b=1, \ldots, B$ bootstrap replicates, constructing $B$ trees. Given a set of covariates $\boldsymbol{x}, \boldsymbol{w}$, the algorithm evaluates $\alpha_{i j, b}(\boldsymbol{x}, \boldsymbol{w})$, which is equal to one of aforementioned proportions if $\boldsymbol{x}, \boldsymbol{w}$ belongs to the same leaf node as data point $i j$ and 0 otherwise, for each bootstrap replicate. Finally, it computes the weights $\alpha_{i j}(\mathbf{x}, \mathbf{w})$ as $\alpha_{i j}(\boldsymbol{x}, \boldsymbol{w})=\sum_{b=1}^{B} \alpha_{i j, b}(\boldsymbol{x}, \boldsymbol{w}) / B$. We remark that we can use the above recursive partitioning algorithm with bootstrap replicates to estimate the leave-one-out estimates $\hat{e}^{-i j}(\boldsymbol{x}, \boldsymbol{w})$ and $\hat{m}^{-i j}(\boldsymbol{x}, \boldsymbol{w})$. For the interested reader, the entire procedure is implemented in the grf package in $\mathrm{R}$ ( $\mathrm{R}$ Core Team, 2020); see Tibshirani et al. (2019) for a detailed vignette.

Compared to parametric approaches in Section 3.2, Causal Forests is a nonparametric estimator that, under some assumptions, achieves consistency and asymptotic convergence to a pivotal Gaussian distribution. The latter is crucial as it allows for construction of p-values and confidence intervals. Unfortunately, these statistical properties only hold if the data is generated in an i.i.d. fashion. However, Causal Forests can be fine-tuned by changing various parameters such as cluster labels, the minimum node size of each tree, and a penalty for imbalanced splits, and we will modify these parameters to improve its performance in clustered data. Specifically, we study three modifications to Causal Forests:

1. The first modification forces Causal Forests to use multilevel propensity scores discussed in Section 3.1 by the tuning parameter W.hat in the grf package. In other words, the estimator $\hat{e}^{(-i j)}(\boldsymbol{x}, \boldsymbol{w})$ in (5) is based on a multilevel logistic regression model with random effect terms instead of the default regression forests estimator based on the aforementioned recursive partitioning algorithm. This modification allows Causal Forests to recognize clustering/hierarchical structure through the multilevel propensity score and adjusts its estimate of $\hat{\tau}(\boldsymbol{x}, \boldsymbol{w})$ accordingly. The modification of this type is denoted as (Est.PS) in subsequent sections.

2. The second modification follows a suggestion from Athey and Wager (2019) where we add cluster label information to define clusters and let Causal Forests "figure out" the rest of the clustering structure from the raw cluster labels; in software, this is achieved by using the parameter clusters in the grf package. The modification forces Causal Forests to draw random subsample of clusters (instead of individuals) and then run the algorithm above within the subsampled clusters. With this modification, the out-of-bag samples are defined as samples that are not in the random subsample of clusters drawn to train the tree. The modification of this type is denoted as (ID) in subsequent sections.

3. The third modification combines the above two modifications and is denoted as (Est.PS+ID) in subsequent sections.

The next sections evaluate these modifications to Causal Forests through an extensive simulation study and a real data analysis.

\section{Simulation Study}

We conducted a large-scale simulation study to assess the performance of methods in Section 3. Our simulation study can be broadly categorized into two designs and is summarized in Table 1. Design 1 assumes a constant treatment effect and generates data from three types of multilevel structures: twolevel, three-level, and cross-classified. Design 2 is limited to two-level structures, but has varying treatment 
effects across clusters and mis-specifies the outcome and selection models to test the robustness of methods to model mis-specification. For both designs, we compare the performance between the default Causal Forests without any modifications, the modified Causal Forests discussed above, and traditional multilevel propensity score and DR methods discussed in Sections 3.1 and 3.2. In particular, under Design 1, the IPW estimator, MMW-S estimator, and two DR estimators use correctly specified propensity score and outcome models and represent an ideal scenario whereby a careful investigator was nearly or completely successful in modeling. Based on asymptotic theory, the DR estimators should perform best under Design 1. However, under Design 2, these four estimators use mis-specified propensity score and outcome models with varying degrees of the model mis-specification and represent a more realistic scenario whereby a careful investigator, despite his/her best efforts, was partially successful in modeling. Under Design 2, nonparametric methods like Causal Forests have the potential to show more promise in achieving better performance than traditional methods since ML methods flexibly and (nearly) automatically capture local structure and model a wider array of functional forms, including those that are used in traditional DR and non-DR methods.

Table 1: A Summary of Simulation Designs 1 and 2

\begin{tabular}{lll}
\hline & Design 1 & Design 2 \\
\hline Clustering structure & two-level & two-level \\
& three-level & \\
& cross-classified & \\
Treatment effects & constant & cluster-specific \\
Model complexity & main effects & main effects and interactions \\
Model specification & correct & incorrect \\
\hline
\end{tabular}

For all simulation designs, we repeated the simulation 1000 times. We evaluated the performance of each estimator by measuring the absolute relative bias (\%), standard deviation (SD), and mean squared error (MSE) defined as follows.

$$
\begin{aligned}
\operatorname{Bias}(\%) & =100 \times\left|\frac{1}{M} \sum_{m=1}^{1000} \frac{\left(\hat{\tau}_{m}-\tau\right)}{\tau}\right| \\
\mathrm{SD} & =\sqrt{\frac{1}{M-1} \sum_{m=1}^{1000}\left(\hat{\tau}_{m}-\overline{\hat{\tau}}\right)^{2}} \\
\mathrm{MSE} & =\frac{1}{M} \sum_{m=1}^{1000}\left(\hat{\tau}_{m}-\tau\right)^{2}
\end{aligned}
$$

Here, $\hat{\tau}_{m}, m=1, \ldots, 1000$ is the $m$-th estimate of the ATE from 1000 simulations. Computer code for the simulation study is available in the supplemental materials.

\subsection{Design 1 with Two-level Data}

The data generating model for two-level clustered data is stated below. The specific parameter values in the model were based on our empirical data from TIMSS.

1. For each cluster $j=1, \ldots, J$, generate the total number of individuals in each cluster $n_{j}$ by drawing a number from a normal distribution with mean $I$ and standard deviation $s d$ and rounding it to the nearest integer.

2. For each individual $i=1, \ldots, n_{j}$ in cluster $j$, generate cluster-level and individual-level covariates 
$\boldsymbol{W}_{j}=\left(W_{1 j}, W_{2 j}\right)$ and $\boldsymbol{X}_{i j}=\left(X_{1 i j}, X_{2 i j}\right)$ as follows.

$$
\begin{aligned}
& \left(\begin{array}{l}
W_{1 j} \\
W_{2 j}
\end{array}\right) \sim N\left[\left(\begin{array}{l}
0 \\
0
\end{array}\right), \quad\left(\begin{array}{cc}
2 & .2 \\
.2 & 2
\end{array}\right)\right] \\
& \left(\begin{array}{l}
X_{1 i j} \\
X_{2 i j}
\end{array}\right) \sim N\left[\left(\begin{array}{l}
0.1 W_{1 j}+0.05 W_{2 j}+\kappa_{1 j} \\
0.08 W_{1 j}+0.1 W_{2 j}+\kappa_{2 j}
\end{array}\right), \quad\left(\begin{array}{cc}
10 & 2 \\
2 & 15
\end{array}\right)\right] \\
& \left(\begin{array}{l}
\kappa_{1 j} \\
\kappa_{2 j}
\end{array}\right) \sim N\left[\left(\begin{array}{l}
0 \\
0
\end{array}\right), \quad\left(\begin{array}{cc}
1 & 0.1 \\
0.1 & 1
\end{array}\right)\right]
\end{aligned}
$$

Individual-level covariates are functions of cluster-level covariates $W_{1 j}, W_{2 j}$ and random errors $\kappa_{1 j}, \kappa_{2 j}$. This type of model for the covariates reflects studies where cluster-level school characteristics impact students' malleable characteristics. For example, it is plausible that students' classroom behavior, motivation, grit, and/or goal orientations (i.e., individual-level covariates $\boldsymbol{X}_{i j}$ ) are affected by location of the school, school funding, schools' vision or climate/culture (i.e., cluster-level covariates $\boldsymbol{W}_{j}$ ). We remark that depending on the study at hand, $\boldsymbol{X}_{i j}$ and $\boldsymbol{W}_{j}$ can have different relationships, such as $\boldsymbol{X}_{i j}$ and $\boldsymbol{W}_{j}$ being independent of each other or $\boldsymbol{W}_{j}$ being affected by $\boldsymbol{X}_{i j}$.

3. Generate individual treatment status $Z_{i j}$ from the following random-effects logistic propensity score model.

$$
\begin{aligned}
\operatorname{logit}\left(\pi_{i j}\right) & =0+0.1 X_{1 i j}+0.03 X_{2 i j}+0.16 W_{1 j}+0.08 W_{2 j}+R_{j}, \quad R_{j} \sim N(0,1) \\
Z_{i j} & \sim \operatorname{Bernoulli}\left(\pi_{i j}\right)
\end{aligned}
$$

where $\pi_{i j}$ is the propensity score for individual $i$ in cluster $j$, and $R_{j}$ is the random effect for cluster $j$ with mean of 0 and variance of 1 . The intra-class correlation (ICC) is around 0.23.

4. Generate the potential outcomes $Y_{i j}(1), Y_{i j}(0)$ and observed outcome $Y_{i j}$ from a random-effects linear regression model.

$$
\begin{aligned}
Y_{i j}(z) & =100+\tau \cdot z+2 X_{1 i j}+1 X_{2 i j}+2 W_{1 j}+1.5 W_{2 j}+U_{j}+\epsilon_{i j} \\
Y_{i j} & =Z_{i j} Y_{i j}(1)+\left(1-Z_{i j}\right) Y_{i j}(0) \\
U_{j} & \sim N(0,10), \epsilon_{i j} \sim N(0,100)
\end{aligned}
$$

Here, $U_{j}$ is the random effect for cluster $j$ with mean of 0 and variance of 10 , and $\epsilon_{i j}$ is the random error for individual $i$ in cluster $j$ with mean of 0 and variance of 100. Also, the treatment effect is constant and set to $\tau=2$. The ICC is 0.1 .

Figure 1 summarizes the results. Each row category, denoted by $\left(\mathrm{J}, \mathrm{I}_{(s d)}\right)$, represents three sub-types of two-level data defined by the number of clusters $J$ and the mean size of each cluster $I$ along with its standard deviation $s d$. For example, the first row category is a two-level dataset with $J=150$ clusters and each cluster has, on average, 30 individuals with standard deviation of 2 . In this condition, we observed that the two DR estimators had the smallest bias and MSE, and the MMW-S estimator had the largest bias and MSE. The performance of the MMW-S estimator was surprising given that it is the de-facto estimator for the ATE in multilevel data. In Appendix C, we computed MMW-S across different choices of $S$ and show that MMW-S is sensitive to the choice of $S$, and the advice from Hong (2010) about choosing $S$ may not be always ideal in our setup. Between Causal Forests and traditional methods, the performance of modified Causal Forests with multilevel propensity scores (CF+Est.PS) generally lied somewhere in between DR estimators and non-DR estimators in terms of bias and MSE. But we observed the modified Causal Forests using multilevel propensity scores had the smallest MSE across all estimators when the mean size of clusters was greater than the number of clusters. Among Causal Forests with modifications, the modified Causal Forests using multilevel propensity scores had the smallest bias and variance. Also, providing explicit cluster labels $(\mathrm{CF}+\mathrm{ID})$ did not improve the performance of Causal Forests. For instance, when the mean cluster size was larger than the number of clusters, Causal Forests with only cluster labels showed the worst performance across all estimators. However, Causal Forests using both multilevel propensity scores and cluster labels performed similarly to Causal Forests using multilevel propensity scores.

Intrigued by the poor performance of the modified Causal Forests based only on cluster labels, Figure 2 explores the properties of Causal Forests when we increase the sample size beyond what is typical in most education studies. For better visualization, Figure 2 trims the top and bottom $10 \%$ of estimates from 


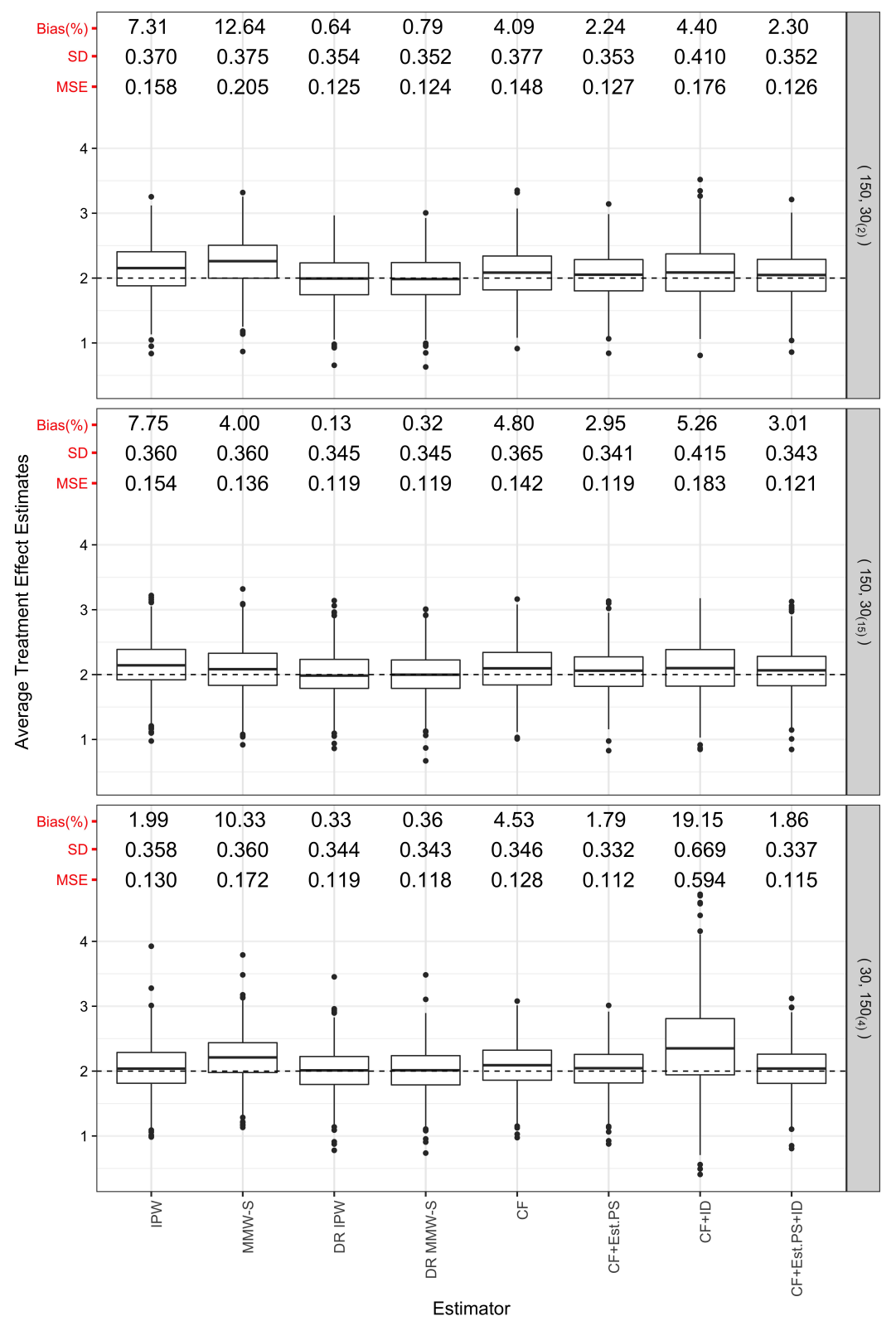

Figure 1: Performance of ATE estimates in two-level data under Design 1. $\left(J, \mathrm{I}_{(s d)}\right)$ represent the number of clusters and the mean size of clusters and its standard deviation, respectively. IPW represents inverse-propensity weighting and MMW-S represents marginal mean weighting through stratification. DR IPW and DR MMW-S represent doubly robust estimators with IPW and MMW-S weights, respectively. CF represents Causal Forests without modification. $\mathrm{CF}+$ Est.PS represents $\mathrm{CF}$ with propensity scores from a multilevel logistic regression. $\mathrm{CF}+\mathrm{ID}$ represents $\mathrm{CF}$ with cluster labels. $\mathrm{CF}+$ Est.PS $+\mathrm{ID}$ represents $\mathrm{CF}$ with propensity scores from a multilevel logistic regression and cluster labels. The dashed black line indicates the true average treatment effect value of 2 .

modified Causal Forests using only cluster labels in the $\left(\mathrm{J}, \mathrm{I}_{(s d)}\right)=\left(10,5000_{(4)}\right)$ setting. But the absolute relative bias, SD, and MSE are computed using all 1000 estimates from the simulation. Interestingly, when the number of clusters was 5000 and the size of each cluster was around 10, the default Causal Forests and modified Causal Forests with cluster labels performed best in terms of MSE among all Causal Forests methods. On the other hand, when the number of clusters was 10 and the size of each cluster was around 5000, Causal Forests with estimated propensity scores performed best in terms of bias and MSE among all Causal Forests methods; in fact, Causal Forests with cluster labels performed worse than the 
default Causal Forests.

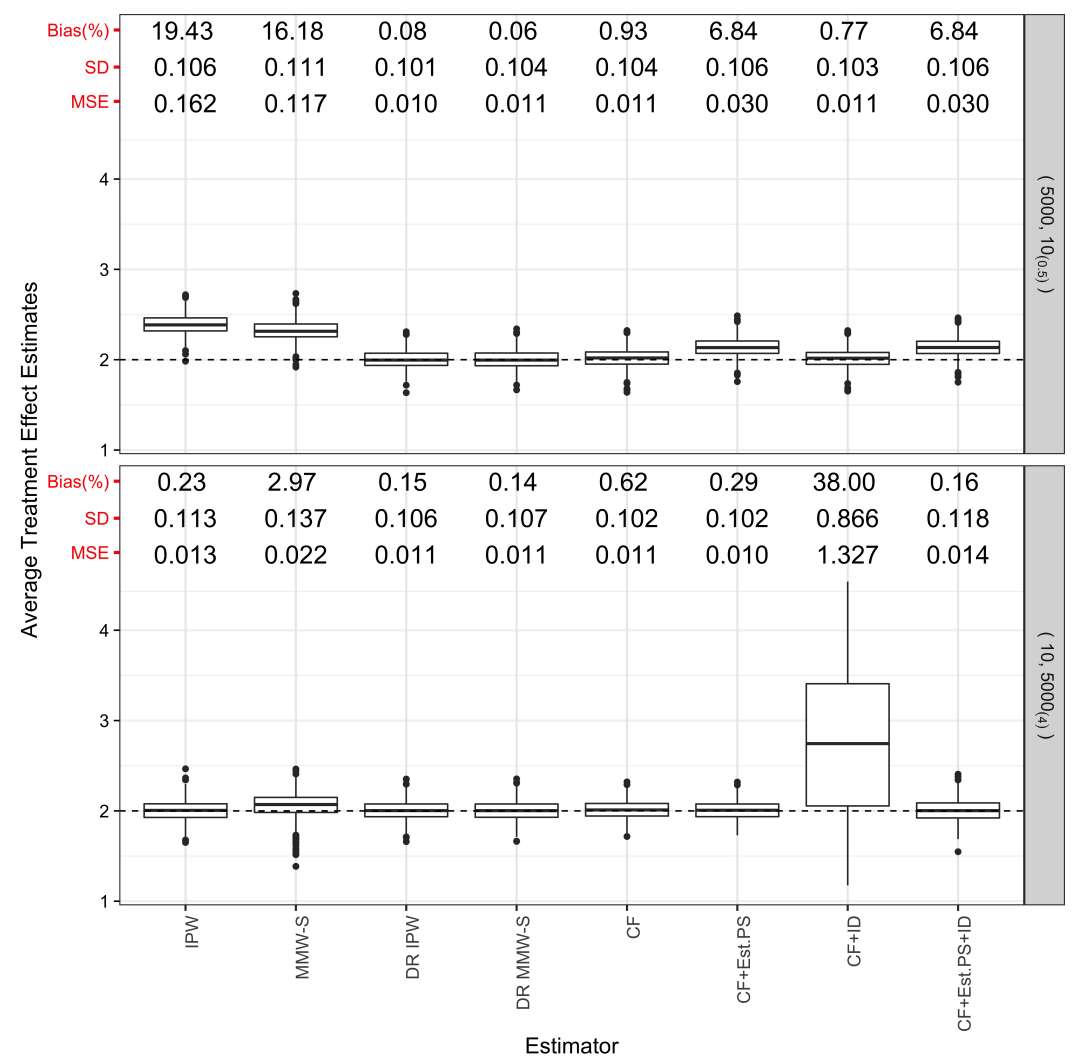

Figure 2: Performance of ATE estimates in two-level data: asymptotic properties. $\left(J, \mathrm{I}_{(s d)}\right)$ represent the number of clusters and the mean size of clusters and its standard deviation, respectively. IPW represents inverse-propensity weighting and MMW-S represents marginal mean weighting through stratification. DR IPW and DR MMW-S represent doubly robust estimators with IPW and MMW-S weights, respectively. CF represents Causal Forests without modification. $\mathrm{CF}+$ Est.PS represents $\mathrm{CF}$ with propensity scores from a multilevel logistic regression. $\mathrm{CF}+\mathrm{ID}$ represents $\mathrm{CF}$ with cluster labels. $\mathrm{CF}+$ Est.PS $+\mathrm{ID}$ represents $\mathrm{CF}$ with propensity scores from a multilevel logistic regression and cluster labels. The dashed black line indicates the true average treatment effect value of 2 .

The result of Figure 2 agrees with some prior theoretical results concerning clustered data and the underlying sampling splitting procedure behind Causal Forests. For example, prior works in econometrics have shown that estimators for cluster-robust standard errors are generally consistent if the number of clusters goes to infinity and the size of the cluster is relatively small in comparison (Wooldridge, 2010). Additionally, in clustered randomized trials where the treatment is assigned at the cluster level, many methods typically require the number of clusters to be much larger than the size of the clusters to achieve consistent and asymptotically Normal estimates of the ATE; see (Donner \& Klar, 2010), (Hayes \& Moulton, 2009), (Kang \& Keele, 2018) and references therein. Finally, as mentioned in Section 3.3, when Causal Forests is provided with cluster labels, the underlying sample splitting procedure is done at the cluster level. This means that for a small number of clusters, there will be fewer clusters to train the tree, potentially leading to large biases. In contrast, for a large number of clusters, there will be many clusters to train the tree, leading to smaller biases. Combining these insights with Figure 2, in two-level data, we believe that providing cluster labels to Causal Forests is only useful in a setting where the number of clusters is much larger than the size of clusters.

\subsection{Design 1 with Three-Level Data}

Three-level data were generated in a similar way as two-level data, but with an additional set of twodimensional continuous covariates at the highest hierarchy. Specifically, we varied the number of highest- 
level clusters $K$ and the number of intermediate-level clusters where the latter was determined by drawing a number from a normal distribution with mean $J$ and standard deviation $s d$ and rounding it to the nearest integer. Also, the propensity score and outcome models for three-level data were:

$$
\begin{aligned}
\operatorname{logit}\left(\pi_{i j k}\right)= & -0.2+0.1 X_{1 i j k}+0.03 X_{2 i j k}+0.1 W_{1 j k}+0.08 W_{2 j k}+0.1 Q_{1 k}+0.05 Q_{2 k}+R_{j k}^{W}+R_{j}^{Q} \\
Y_{i j k}(z)= & 100+2 \cdot z+2 X_{1 i j k}+1 X_{2 i j k}+2 W_{1 j k}+1.5 W_{2 j k}+1 Q_{1 k}+0.5 Q_{2 k}+U_{j k}^{W}+U_{k}^{Q}+\epsilon_{i j k} \\
& R_{j k}^{W} \sim N(0,1), R_{k}^{Q} \sim N(0,1), U_{j k}^{W} \sim N(0,10), U_{k}^{Q} \sim N(0,7), \epsilon_{i j k} \sim N(0,100)
\end{aligned}
$$

We highlight three additional differences between the three-level simulation design and the two-level simulation design in Section 4.1. First, there are two more continuous covariates, $Q_{1 k}, Q_{2 k}$, at the highest level of the hierarchy. Second, there are two random effect terms in the propensity score model (i.e., $R_{j k}^{W}$ and $R_{j}^{Q}$ ) and in the outcome model (i.e., $U_{j k}^{W}$ and $U_{k}^{Q}$ ). Random effect terms follow a Normal distribution with mean zero and variance $\sigma^{2}$. The additional random effect term in each model corresponds to the additional clustering effect at the highest level of the hierarchy. The ICCs for $R_{j k}^{W}$ and $R_{j}^{Q}$ in the propensity score model are around 0.19 and 0.19 , respectively, and the ICCs for $U_{j k}^{W}$ and $U_{k}^{Q}$ in the outcome model are 0.1 and 0.07 , respectively. Third, we provide either intermediate-level or highest-level cluster labels when we modify Causal Forests. Additional details on generating three-level data are in Appendix A.

Figure 3 shows the simulation results for three-level data. Similar to the result from two-level data, the two DR estimators had the smallest bias. However, unlike the results from two-level data, modified Causal Forests with multilevel propensity scores was able to achieve the smallest MSE among all estimators; their MSEs were equal to or slightly lower than the MSEs of the DR estimators. The bias of modified Causal Forests with multilevel propensity scores was in between the bias of the non-DR estimators and the DR estimators; the notable exception is the MMW-S estimator with 15 three-level clusters where its bias was lower than modified Causal Forests with multilevel propensity scores. Also, similar to the result from two-level data, among different modifications to Causal Forests, we found that including the multilevel propensity scores had the largest improvement in terms of bias and variance compared to including only cluster labels into Causal Forests or using the default Causal Forests. Among Causal Forests estimators with only cluster labels, including the intermediate-level cluster labels $(\mathrm{CF}+\mathrm{ID} 2)$ provided more bias and variance reduction than including the highest-level cluster labels $(\mathrm{CF}+\mathrm{ID} 3)$. However, as seen from the two-level setting, using only cluster labels did not provide significant benefits compared to only using multilevel propensity scores, and there were no additional gains in performance from including the cluster labels into modified Causal Forests already using multilevel propensity scores.

\subsection{Design 1 with Cross-classified Data}

Our data generating model for cross-classified data follows closely to Meyers and Beretvas (2006) where we assume that cross-factor residuals are uncorrelated and there are three feeders from one factor to the other factor. The two factors' numbers, denoted as F1 and F2, varied simultaneously. The exact propensity score and outcome models in cross-classified data were:

$$
\begin{aligned}
\operatorname{logit}\left(\pi_{i j k}\right)= & -0.2+0.1 X_{1 i(j k)}+0.03 X_{2 i(j k)}+0.1 W_{1 j}+0.08 W_{2 j}+0.1 Q_{1 k}+0.05 Q_{2 k}+R_{j}^{W}+R_{k}^{Q} \\
Y_{i(j k)}(z)= & 100+2 \cdot z+2 X_{1 i(j k)}+1 X_{2 i(j k)}+2 W_{1 j}+1.5 W_{2 j}+1 Q_{1 k}+0.5 Q_{2 k}+U_{j}^{W}+U_{k}^{Q}+\epsilon_{i(j k)} \\
& R_{j}^{W} \sim N(0,1), R_{k}^{Q} \sim N(0,0.5), U_{j}^{W} \sim N(0,10), U_{k}^{Q} \sim N(0,7), \epsilon_{i j k} \sim N(0,100)
\end{aligned}
$$

Compared to two-level data, in cross-classified data, there are two more continuous covariates to indicate an additional cluster level, $Q_{1 k}, Q_{2 k}$. Also, both the propensity score and the outcome models have two random effect terms for the two factors (i.e., $R_{j}^{W}, R_{k}^{Q}$ and $U_{j}^{W}, U_{k}^{Q}$ ). Random effect terms follow a Normal distribution with mean zero and variance $\sigma^{2}$. The ICCs for $R_{j}^{W}$ and $R_{k}^{Q}$ in the propensity score model are around 0.21 and 0.10, respectively, and the ICCs for $U_{j}^{W}$ and $U_{k}^{Q}$ in the outcome model are 0.1 and 0.07 , respectively. Finally, for modified Causal Forests based on cluster labels, we use either the first, second, or combined factor labels. Additional details on generating cross-classified data are in Appendix B.

Figure 4 provides the results under cross-classified data setting. While the two DR estimators had the smallest bias in most scenarios, the modified Causal Forests with multilevel propensity scores had the smallest bias and MSE when the cluster size (here, factor 1 clusters) was larger than the number of the clusters. In the opposite setting where the size of the clusters was smaller than the number of clusters, the bias and MSE of modified Causal Forests with multilevel propensity scores was somewhere in between 


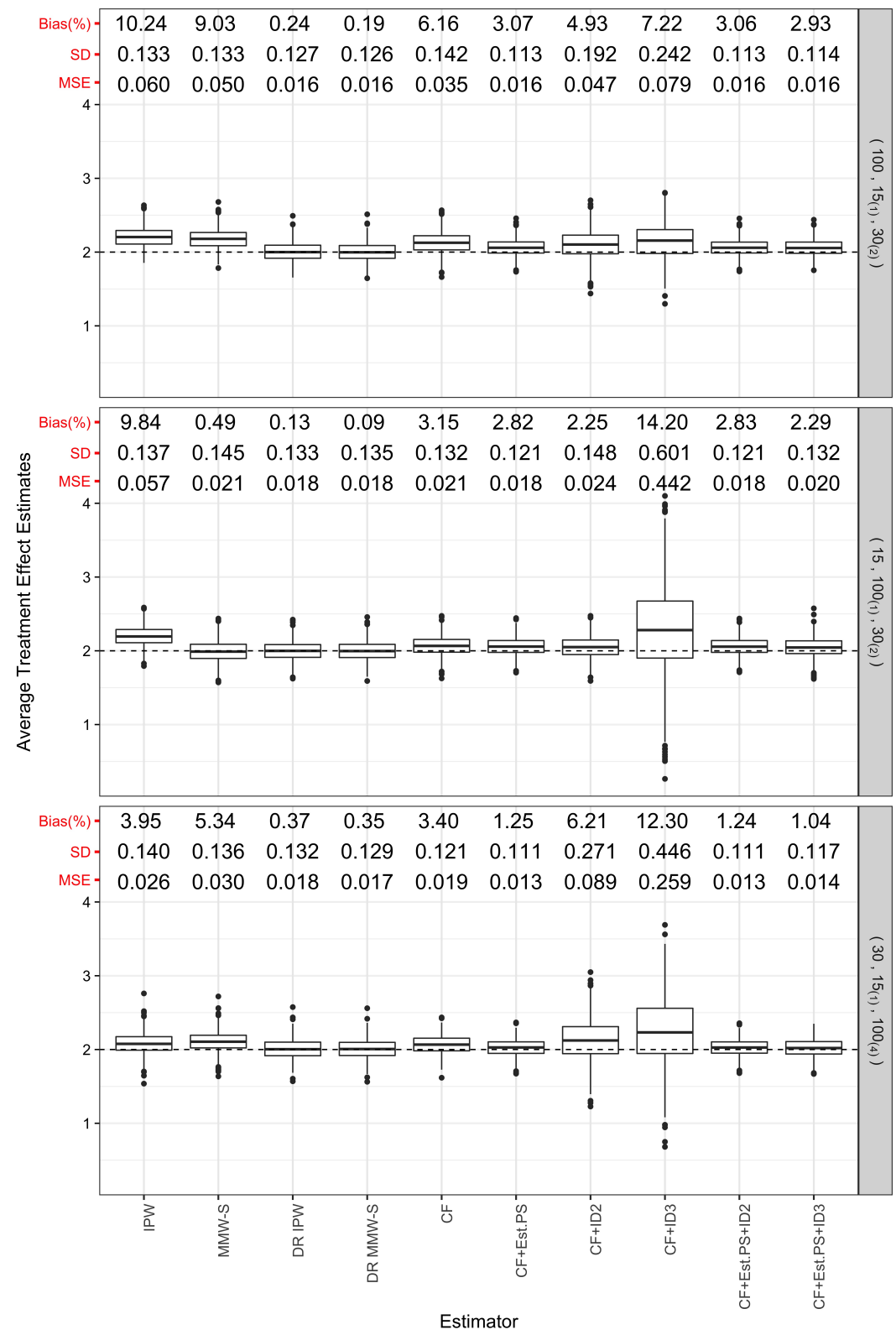

Figure 3: Performance of ATE estimates in three-level data. $\left(K, \mathrm{~J}_{(s d)}\right.$, and $\left.\mathrm{I}_{(s d)}\right)$ represent the number of level-3 clusters, the mean size of level-3 clusters with its standard deviation, and the mean size of level-2 clusters with its standard deviation, respectively. IPW represents inversepropensity weighting and MMW-S represents marginal mean weighting through stratification. DR IPW and DR MMW-S represent doubly robust estimators with IPW and MMW-S weights, respectively. CF represents Causal Forests without modification. CF+Est.PS represents CF with propensity scores from a multilevel logistic regression. $\mathrm{CF}+\mathrm{ID} 2$ and $\mathrm{CF}+\mathrm{ID} 3$ represent $\mathrm{CF}$ with level-2 cluster labels and level-3 cluster labels, respectively. $\mathrm{CF}+\mathrm{Est} . \mathrm{PS}+\mathrm{ID} 2$ and $\mathrm{CF}+$ Est.PS $+\mathrm{ID} 3$ represent $\mathrm{CF}$ with propensity scores from a multilevel logistic regression and level-2 or level-3 cluster labels. The dashed black line indicates the true average treatment effect value of 2 .

those from DR and non-DR estimators. The bias of modified Causal Forests with only cluster labels was comparable to or sometimes worse than that of the IPW estimator or MMW-S estimator. Among modified Causal Forests using cluster labels, Causal Forests using combined cluster identifiers (CF+F12ID) had the smallest MSE than those using only one factor identifier ( $\mathrm{CF}+\mathrm{F} 1 \mathrm{ID}$ or $\mathrm{CF}+\mathrm{F} 2 \mathrm{ID})$. Similar to twolevel and three-level settings, having both estimated propensity scores and cluster IDs in Causal Forests 
provided no additional benefits compared to having only multilevel propensity scores.

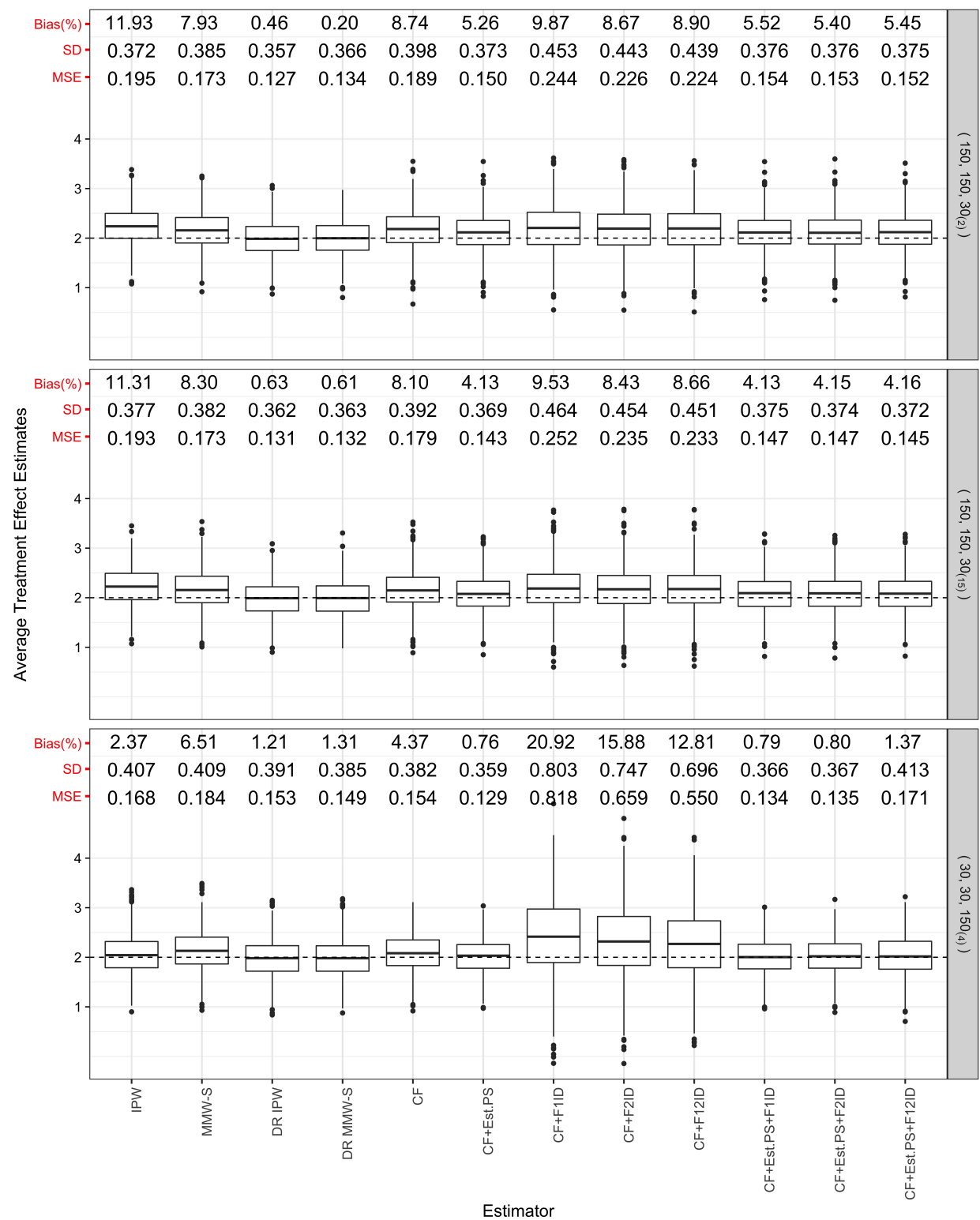

Figure 4: Performance of ATE estimates in cross-classified data. (F1, F2, and $\left.\mathrm{I}_{(s d)}\right)$ represent the number of factor- 1 clusters, the number of factor- 2 clusters, and the mean size of factor- 1 clusters with its standard deviation, respectively. IPW represents inverse-propensity weighting and MMW-S represents marginal mean weighting through stratification. DR IPW and DR MMW-S represent doubly robust estimators with IPW and MMW-S weights, respectively. CF represents Causal Forests without modification. CF+Est.PS represents CF with propensity scores from a multilevel logistic regression. $\mathrm{CF}+\mathrm{F} 1 \mathrm{ID}, \mathrm{CF}+\mathrm{F} 2 \mathrm{ID}$, and $\mathrm{CF}+\mathrm{F} 12 \mathrm{ID}$ represent $\mathrm{CF}$ with the first factor labels, the second factor labels, and the two factors' combined labels, respectively. $\mathrm{CF}+$ Est.PS $+\mathrm{F} 1 \mathrm{ID}, \mathrm{CF}+$ Est.PS $+\mathrm{F} 2 \mathrm{ID}$, and $\mathrm{CF}+$ Est.PS $+\mathrm{F} 12 \mathrm{ID}$ represent $\mathrm{CF}$ with propensity scores from a multilevel logistic regression and the first factor, the second factor, or the combined labels. The dashed black line indicates the true average treatment effect value of 2 . 


\subsection{Design 2 with Two-level Data}

In Design 2, we generated two-level data similar to Design 1, except we changed the propensity score and the outcome model as follows:

$$
\begin{aligned}
\operatorname{logit}\left(\pi_{i j}\right)= & 0+0.1 X_{1 i j}+0.03 X_{2 i j}+0.16 W_{1 j}+0.08 W_{2 j}+\beta_{1} X_{1 i j} W_{2 j}+R_{j} \\
Y_{i j}(z)= & 100+\tau \cdot z+2 X_{1 i j}+1 X_{2 i j}+2 W_{1 j}+0.5 z \cdot W_{1 j}+1.5 W_{2 j}+\beta_{2} X_{1 i j} W_{2 j}+U_{j}+\epsilon_{i j} \\
& \boldsymbol{\beta}=\left(\beta_{1}, \beta_{2}\right) \in\{(0.02,0.3),(0.04,0.6),(0.06,1)\} \\
& R_{j} \sim N(0,1), U_{j} \sim N(0,10), \epsilon_{i j} \sim N(0,100)
\end{aligned}
$$

There are two major differences between the previous two-level simulation design in Section 4.1 and the new two-level simulation design. First, there is now an interaction term between the individual-level covariate $X_{1 i j}$ and the cluster-level covariate $W_{2 j}$ in both the propensity score and outcome models. The interaction term's magnitude is controlled by parameter $\boldsymbol{\beta}$. We use this extra interaction term to intentionally mis-specify the propensity score and the outcome model by only fitting the main effects; note that this type of mis-specification can also be seen as a form of omitted variable bias where we "omitted" the interaction terms. Second, in the outcome model, there is an interaction term between the treatment $z$ and the cluster-level covariate $W_{1 j}$. This interaction term creates heterogeneous cluster-specific treatment effects where the cluster-specific CATE is $\tau+0.5 W_{1 j}$. However, the mean of $W_{1 j}$ is zero and hence, the overall ATE remains the same as before, $\tau=2$.

Figure 5 summarizes the results with different values of $\boldsymbol{\beta}$. As the propensity score and outcome models became more mis-specified, the DR estimators behaved similarly to non-DR estimators and both were equally biased. When the model mis-specification was moderate to large, Causal Forests methods almost always performed better in terms of bias and MSE than any of the traditional methods (IPW, MMW-S, and DR estimators) across different conditions on the number of clusters and the mean size of clusters. Even the default Causal Forests that did not incorporate any clustering information did better than traditional methods that incorporated clustering. This suggests that when parametric models are misspecified, nonparametric methods like Causal Forests perform better than traditional parametric methods, even if the ML methods were not initially designed for clustered data, because they can automatically capture the omitted interaction terms that potentially lead to more significant bias compared to bias arising from ignoring clustering structure inside ML methods. Also, the modified Causal Forests with multilevel propensity scores outperformed the default Causal Forests in terms of variance across all settings, and they did better than the default Causal Forests in terms of MSE when the model mis-specification was small to moderate. Finally, Causal Forests using only cluster labels generally performed worse than Causal Forests using multilevel propensity scores.

\subsection{Takeaways from Simulation Studies}

Overall, the simulation studies above suggest some guidelines on how to modify ML methods for clustered/hierarchical data. While these are not meant to encompass every ML method in causal inference or every possible type of clustered data in practice, we hope that the suggestions provided below based our simulation study with Causal Forests can serve as useful guidelines for empirical or theoretical analyses of clustered data with ML methods.

1. For Causal Forests, incorporating multilevel, hierarchical structure through multilevel propensity score models typically had the largest improvement in terms of bias and MSE compared to Causal Forests that directly used cluster labels. In particular, Causal Forests using cluster labels should only be reserved to settings when the number of clusters is much larger than the size of clusters.

2. In general, there was no significant benefit in terms of bias and MSE when using both multilevel propensity scores and cluster labels inside Causal Forests compared to just using multilevel propensity scores.

3. Causal Forests with multilevel propensity scores almost always performed better in terms of bias and MSE than multilevel propensity score estimators (IPW and MMW-S) with correctly specified parametric propensity scores, but generally performed worse than DR methods with correctly specified parametric propensity score and outcome models, with some notable exceptions. In particular, Causal Forests with multilevel propensity scores had similar or slightly lower MSE than those those from DR methods with correctly specified propensity score and outcome models whenever the size of the clusters is larger than the number of clusters. 


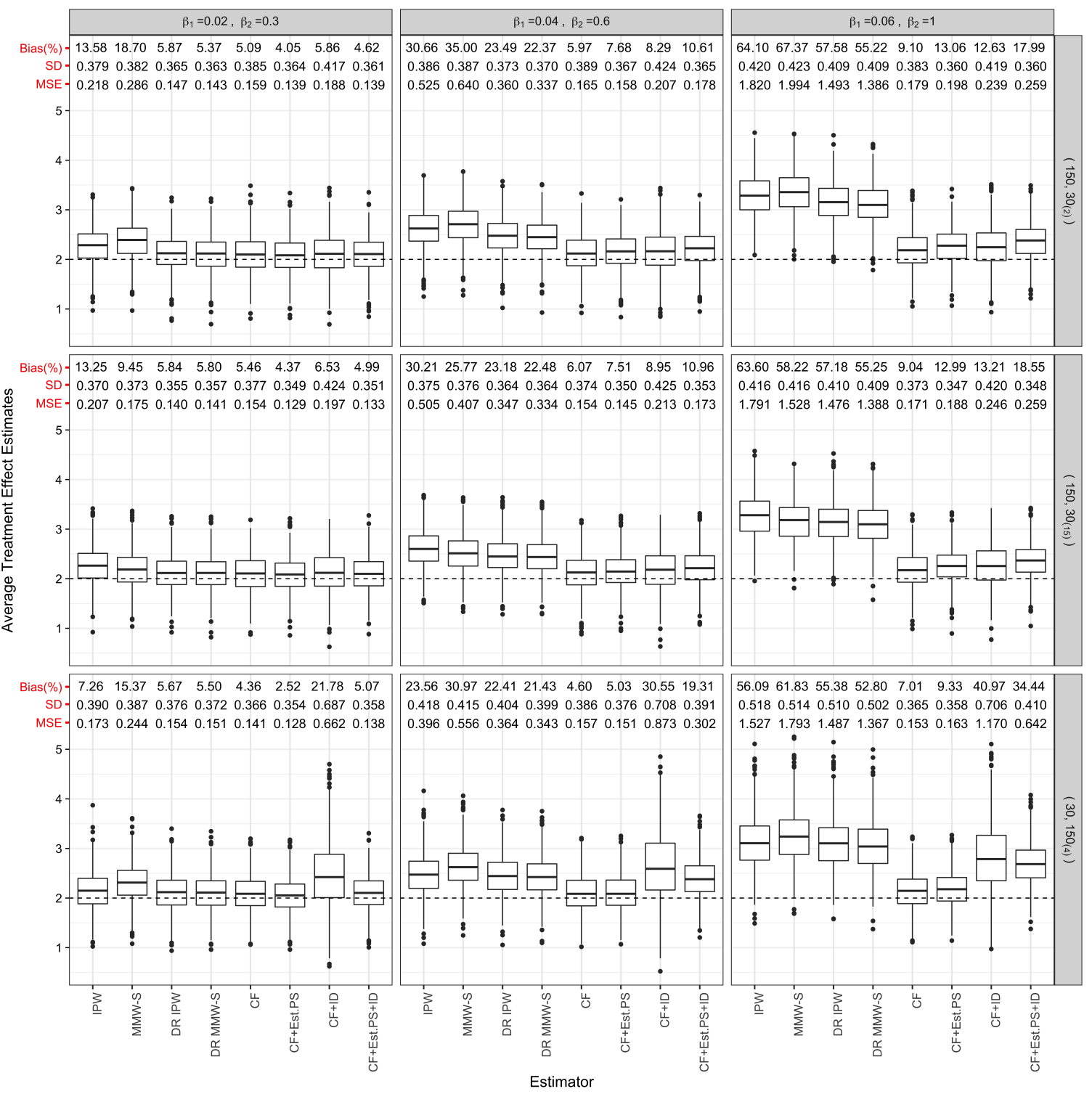

Figure 5: Performance of ATE estimates in two-level data under Design 2; $\beta_{1}$ and $\beta_{2}$ are coefficients of the omitted interaction terms in parametric selection and outcome models, respectively. $\left(J, \mathrm{I}_{(s d)}\right)$ represent the number of clusters and the mean size of clusters and its standard deviation, respectively. IPW represents inverse-propensity weighting and MMW-S represents marginal mean weighting through stratification. DR IPW and DR MMW-S represent doubly robust estimators with IPW and MMW-S weights, respectively. CF represents Causal Forests without modification. CF + Est.PS represents $\mathrm{CF}$ with propensity scores from a multilevel logistic regression. $\mathrm{CF}+\mathrm{ID}$ represents $\mathrm{CF}$ with cluster labels. $\mathrm{CF}+\mathrm{Est} . \mathrm{PS}+\mathrm{ID}$ represents $\mathrm{CF}$ with propensity scores from a multilevel logistic regression and cluster labels. The dashed black line indicates the true average treatment effect value of 2 . 
4. If DR or non-DR estimators were using parametric propensity score or outcome models that were moderately mis-specified, Causal Forests with multilevel propensity scores outperformed them in terms of bias and MSE.

5. Causal Forests with multilevel propensity scores performed better in terms of MSE than the default Causal Forests without any modifications, even if the multilevel propensity score model inside modified Causal Forests was moderately mis-specified.

\section{Real Data Study}

\subsection{Data}

TIMSS is an international educational study about students' achievement progresses in mathematics and science and is sponsored by the International Association for the Evaluation of Educational Achievement (IEA). Since 1995, TIMSS has collected data among students in Grades 4 and 8 every four years. To do this, TIMSS uses a two-stage stratified cluster sampling design where in the first stage, each country selects schools based on important demographic variables (e.g., school location and/or school gender type), and in the second stage, each school randomly selects one or more intact classrooms (Martin, Mullis, \& Hooper, 2016). The most recent completed wave of TIMSS was in 2015 and conducted across 60 countries.

We used the 2015 Korea TIMSS Grade- 8 data to investigate the effect of private math lessons. The data contained 5309 students from 150 middle schools where the school sizes ranged from 6 to 75 students; the mean size of schools was about 30 . While the original data resembled a three-level structure based on a student-class-school hierarchy, we found that most of the schools selected one classroom; 130 schools selected one classroom, and 20 schools selected two classrooms. Since there was a near one-to-one correspondence between school and class levels, we analyzed the data as a two-level data where students are nested within schools.

The treatment was whether students received private math lessons, with 1 indicating that the student did and 0 otherwise; the treatment was assigned at the student level. The outcome was the first plausible value of students' math achievement scores; in the 2015 TIMSS data, each student obtained five plausible values because they took a subset of items from a full battery of assessment items. In addition, we used 12 covariates that were thought to influence the treatment and outcome variables. Six of 12 covariates were student-level covariates and the other six covariates were school-level covariates. Student-level covariates included 1) gender (sexM, male and female), 2) fathers' highest education level (dad.edu, with three levels including no college, college $d a d_{-} c l l$, and don't know $d a d_{-} q$ ), 3) the number of books at home (books25, with two levels defined as more than 25 books or less than or equal to 25 books), 4) the number of home study supports (hspprt, with three levels including neither own room nor Internet connection, one of them hspprt_1, and both hspprt_2), 5) students' confidence in math (M.stuconf, continuous), and 6) value in math (M.value, continuous). School-level covariates included 1) whether the school is gendered (gender.type, with three levels of all-boys, all-girls girlsch, and co-education coedu), 2) the percentage of economically disadvantaged students (pct.disad, with four levels of 0 to $10 \%, 11$ to $25 \%$ disad_11, 26 to $50 \%$ disad_26, and more than 50\% disad_M50), 3) school location (city.size, with four levels of urban city_ $U$, suburban $c i t y \_S u b$, medium size city city_ $M$, and small town), 4) emphasis on academic success (acad.emph, continuous), 5) math instruction affected by resource shortage (M.resshort, continuous), and 6) discipline problems (dscpn, continuous).

We excluded students whose responses were inconsistent with the following two questions regarding their participation in private math lessons: (Q1) whether students received private math lessons and (Q2) for how many months they received these lessons. In particular, we excluded students who answered "Yes" to (Q1) and "did not attend" to (Q2) and students who answered "No" to (Q1) and answered something other than "did not attend" to (Q2). Additionally, we excluded students who were missing 7 out of 12 covariates. The final sample consisted of 4943 students (93.1\% of the original sample) from 149 schools. In the final sample, the outcome's mean was 606.08 and its standard deviation was 84.19 ; its minimum and maximum were 306.66 and 859.86 , respectively. Data analyzed in this study is included in the supplementary materials.

\subsection{Methods}

We followed our simulation study and estimated a joint propensity score model based on random-effects logistic regression with main effect terms. The outcome model (for DR methods) was based on random- 
effects linear regression model with main effect terms. We also implemented Causal Forests as mentioned in Section 4.1. We used grf package to run Causal Forests. For the IPW, MMW-S, DR IPW, and DR MMW-S estimators, we used Ime4 package (Bates, Mächler, Bolker, \& Walker, 2015) to estimate propensity scores and outcome regression models. Standard errors were estimated using cluster bootstrap sampling with 5000 replicates. All analyses were conducted in R. We evaluated covariate balance between treated and untreated units before estimating the ATE. As a rule of thumb, a good balance for a covariate is when its absolute standardized mean difference is smaller than 0.1 and its variance ratio is in between 4/5 and 5/4 (Rubin, 2001; Shadish, Clark, \& Steiner, 2008; Steiner, Cook, Shadish, \& Clark, 2010). For simplicity, we ignored sampling weights that weighed each individual in the data. Since we did not incorporate sampling weights and multiple plausible values, our proposed analysis plan does not generalize to the study population outside of TIMSS.

\subsection{Results}
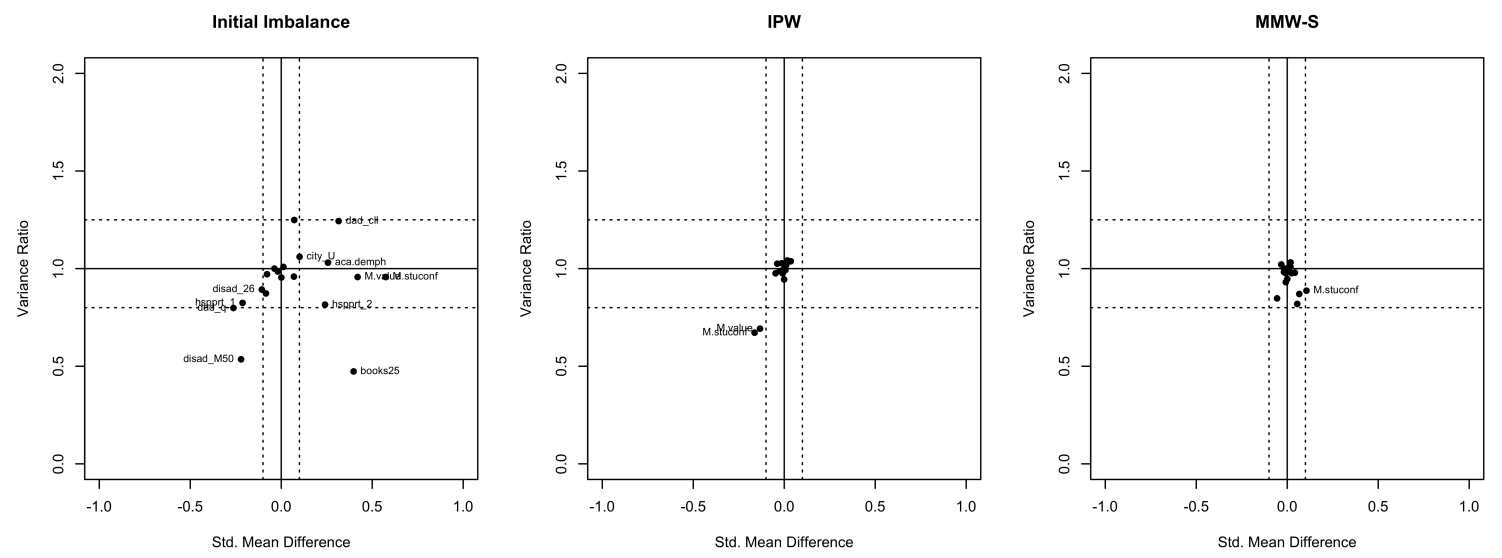

Figure 6: Covariate balance plots before and after propensity score adjustment

Figure 6 provides covariate balance plots before and after propensity score adjustments. Before adjustment, there were imbalances in most of the 12 covariates: dad.edu, books25, hspprt, M.stuconf, M.value, pct.disad, city.size, and acad.emph. After IPW or MMW-S adjustment, we improved balance between private-lessons takers (the treated) and non-takers (the untreated). Weights from MMW-S with 3 strata achieved more successful balance than weights from the IPW estimator; weights from MMW-S achieved almost perfect mean and variance balance, whereas weights from the IPW estimator still left two covariates slightly imbalanced.

Figure 7 summarizes the estimates, standard errors (in parentheses), and 95\% confidence intervals of the ATE using different estimators. The prima facie effect is the unadjusted mean difference in students' math achievement scores between private-lessons takers and non-takers. The unadjusted estimate was 66.88 points and the effect was reduced to 27.67 points and 33.88 points using the IPW estimator and MMW-S estimator, respectively. The DR IPW and DR MMW-S estimators produced estimates of 32.96 and 29.58 points, respectively. The ATE estimates obtained with Causal Forests ranged from 25.54 $(\mathrm{CF}+$ Est.PS $)$ to $30.30(\mathrm{CF}+\mathrm{ID})$. Between Causal Forests, the IPW estimator, and MMW-S estimator, we found that the point estimates of the ATE from Causal Forests using multilevel propensity scores $(\mathrm{CF}+$ Est.PS or CF +Est.PS +ID $)$ were slightly smaller compared to the IPW estimator and MMW-S. Regarding the $95 \%$ confidence intervals of the ATE estimates, we found that all the confidence intervals were overlapping. The IPW estimator yielded the widest confidence interval (i.e., the most conservative standard error), while Causal Forests using multilevel propensity scores $(\mathrm{CF}+$ Est.PS) produced the narrowest confidence interval (i.e., the most liberal standard error). Overall, all estimates reached similar conclusions about the treatment effect, that private math lessons had a positive effect on math achievement scores.

\section{Discussion and Conclusions}

The goal of this paper was to study how to properly modify ML methods originally designed for i.i.d. data to estimate treatment effects in clustered $/$ multilevel data settings. In particular, we explored how 


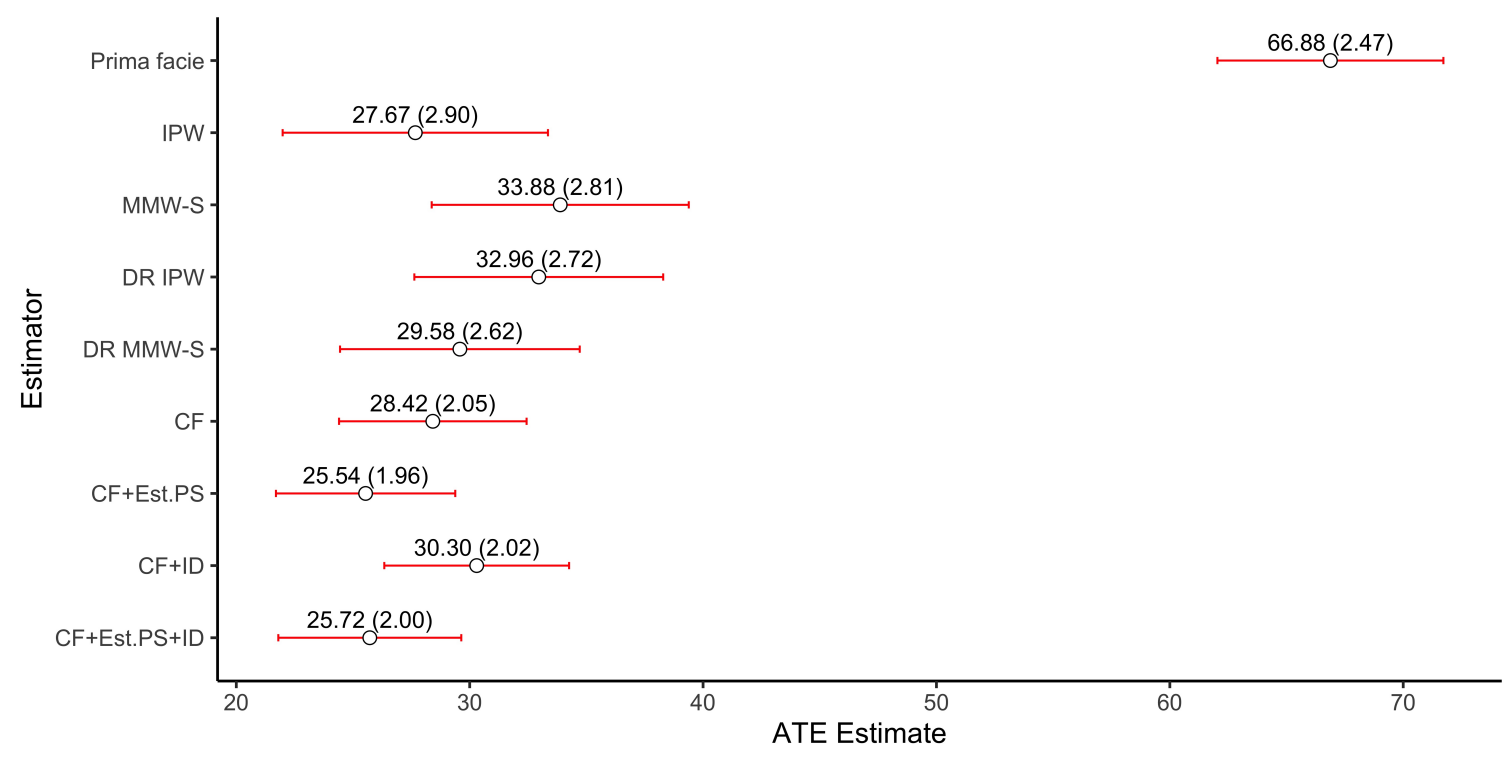

Figure 7: The estimates, standard errors (in parentheses), and 95\% confidence intervals of the ATE of taking private math lessons

to account for cluster or hierarchical structures in Causal Forests by including propensity scores from multilevel logistic regression or cluster labels. As stated in Section 4.5, our simulation studies showed that among all modifications to Causal Forests, using Causal Forests with multilevel propensity scores had the best performance in terms of bias and MSE, and providing cluster labels was only useful in settings where the number of clusters is far larger than the size of the clusters. Also, modified Causal Forests with multilevel propensity scores performed somewhere in between DR and non-DR estimators that had correctly specified propensity score and outcome regression models. However, if these parametric models were mis-specified, modified Causal Forests with multilevel propensity scores performed better than the DR and non-DR estimators in our simulation.

We also conducted a real data study to examine the effects of private math lessons from the 2015 TIMSS data. All methods generally revealed that private math lessons improved math scores, though each estimator exhibited slightly different point estimates. Since each method carries different underlying assumptions about the data generating model, differences in these assumptions likely affected the specific estimated values of the treatment effect. Nevertheless, comparing their results allowed us to assess the plausibility of the underlying assumptions and reinforce our evidence for a causal effect. As such, while Causal Forests with multilevel propensity scores is generally recommended when researchers suspect more complex data generating processes or when there is insufficient subject-matter knowledge to justify parametric models, we recommend using both Causal Forests and traditional DR and non-DR approaches to strengthen the causal conclusion from the study.

There are some limitations of the paper. First, we assumed SUTVA in a clustered setting where the treatment, assigned at the individual level, is hypothesized to not have spillover effects through interference. Second, our simulation study is limited to three types of clustered data structures and we did not analyze more complex clustering structures, such as clustering through spatiotemporal processes. Third, though we explored the consequence of omitting an interaction term when all the confounders were measured in Design 2, we did not consider omitted variable bias arising from unmeasured pre-treatment covariates. Fourth, we primarily used estimated propensity scores from random-effects logistic regression models as part of our modification strategy for Causal Forests. However, it has been shown that fixed effects models with cluster dummies are robust against unmeasured cluster-level variables (Wooldridge, 2010; Arpino \& Mealli, 2011) and it would be interesting to examine whether using multilevel propensity scores with fixed effects inside ML methods can provide additional benefits in estimating the ATE. Fifth, since treatments are often multi-valued rather than binary, future work may extend the applicability of our results to multi-valued treatment settings by utilizing the results in Imbens (2000) and Imai and Van Dyk (2004). Sixth, this paper primarily focuses on estimating the ATE, but we believe that the insights from this work can be used to address challenges in optimal treatment assignment (Kosorok \& Moodie, 2015; Li, Lu, \& Zhou, 2017; Dimakopoulou, Zhou, Athey, \& Imbens, 2017). 
Despite these limitations, this paper provides a simple set of ways to modify pre-existing ML methods in causal inference in order to estimate the ATE in multilevel settings. Our proposed modification of Causal Forests by using propensity scores from multilevel regression models helped minimize bias and MSE compared to directly providing cluster labels or using Causal Forests without any modifications. More broadly, this type of modification via the propensity score can serve as a template to modify a wide variety of ML methods when used in multilevel observational studies.

\section{References}

Arpino, B., \& Cannas, M. (2016). Propensity score matching with clustered data. an application to the estimation of the impact of caesarean section on the apgar score. Statistics in Medicine, 35 (12), 2074-2091. doi: https://doi.org/10.1002/sim.6880

Arpino, B., \& Mealli, F. (2011). The specification of the propensity score in multilevel observational studies. Computational Statistics \& Data Analysis, 55(4), 1770-1780. doi: https://doi.org/10.1016/j.csda.2010.11.008

Athey, S., \& Imbens, G. (2016). Recursive partitioning for heterogeneous causal effects. Proceedings of the National Academy of Sciences, 113(27), 7353-7360. doi: https://doi.org/10.1073/pnas.1510489113

Athey, S., Tibshirani, J., \& Wager, S. (2019). Generalized random forests. The Annals of Statistics, 47(2), 1148-1178. doi: https://doi.org/10.1214/18-AOS1709

Athey, S., \& Wager, S. (2019). Estimating treatment effects with causal forests: An application. arXiv preprint arXiv:1902.07409.

Bates, D., Mächler, M., Bolker, B., \& Walker, S. (2015). Fitting linear mixed-effects models using lme4. Journal of Statistical Software, 67(1), 1-48. doi: 10.18637/jss.v067.i01

Breiman, L., Friedman, J. H., Olshen, R. A., \& Stone, C. J. (1984). Classification and regression trees. Wadsworth.

Carvalho, C., Feller, A., Murray, J., Woody, S., \& Yeager, D. (2019). Assessing treatment effect variation in observational studies: Results from a data challenge. Observational Studies.

Chernozhukov, V., Chetverikov, D., Demirer, M., Duflo, E., Hansen, C., Newey, W., \& Robins, J. (2018). Double/debiased machine learning for treatment and structural parameters. The Econometrics Journal, 21(1), C1-C68. doi: https://doi.org/10.1111/ectj.12097

Dimakopoulou, M., Zhou, Z., Athey, S., \& Imbens, G. (2017). Estimation considerations in contextual bandits. arXiv preprint arXiv:1711.07077.

Donner, A., \& Klar, N. (2010). Design and analysis of cluster randomization trials in health research. New York: Wiley.

Hayes, R. J., \& Moulton, L. H. (2009). Cluster randomised trials. Chapman \& Hall/CRC. doi: https://doi.org/10.1201/9781584888178

Hill, J. L. (2011). Bayesian nonparametric modeling for causal inference. Journal of Computational and Graphical Statistics, 20(1), 217-240. doi: https://doi.org/10.1198/jcgs.2010.08162

Hong, G. (2010). Marginal mean weighting through stratification: adjustment for selection bias in multilevel data. Journal of Educational and Behavioral Statistics, 35(5), 499-531. doi: https://doi.org/10.3102/1076998609359785

Hong, G., \& Hong, Y. (2009). Reading instruction time and homogeneous grouping in kindergarten: An application of marginal mean weighting through stratification. Educational Evaluation and Policy Analysis, 31 (1), 54-81. doi: https://doi.org/10.3102/0162373708328259

Hong, G., \& Raudenbush, S. W. (2006). Evaluating kindergarten retention policy: A case study of causal inference for multilevel observational data. Journal of the American Statistical Association, 101, 901-910. doi: https://doi.org/10.1198/016214506000000447 
Hong, G., \& Raudenbush, S. W. (2013). Heterogeneous agents, social interactions, and causal inference. In S. L. Morgan (Ed.), Handbook of causal analysis for social research (pp. 331-352). Springer. doi: https://doi.org/10.1007/978-94-007-6094-316

Imai, K., \& Van Dyk, D. A. (2004). Causal inference with general treatment regimes: Generalizing the propensity score. Journal of the American Statistical Association, 99(467), 854-866. doi: https://doi.org/10.1198/016214504000001187

Imbens, G. W. (2000). The role of the propensity score in estimating dose-response functions. Biometrika, 87(3), 706-710. doi: https://doi.org/10.1093/biomet/87.3.706

Kang, H., \& Keele, L. (2018). Estimation methods for cluster randomized trials with noncompliance: A study of a biometric smartcard payment system in india. arXiv preprint arXiv:1805.03744.

Keller, B., Kim, J.-S., \& Steiner, P. M. (2015). Neural networks for propensity score estimation: Simulation results and recommendations. In L. A. van der Ark, D. M. Bolt, W.-C. Wang, J. A. Douglas, \& M. Wiberg (Eds.), Quantitative psychology research: The 80th annual meeting of the psychometric society (pp. 279-291). Springer. doi: https://doi.org/10.1007/978-3-319-19977-1 ${ }_{2} 0$

Kim, J., \& Seltzer, M. (2007). Causal inference in multilevel settings in which selection processes vary across schools. cse technical report 708. National Center for Research on Evaluation, Standards, and Student Testing (CRESST). doi: https://doi.org/10.1037/e644002011-001

Kim, J.-S., \& Steiner, P. M. (2015). Multilevel propensity score methods for estimating causal effects: A latent class modeling strategy. In L. A. van der Ark, D. M. Bolt, W.-C. Wang, J. A. Douglas, \& M. Wiberg (Eds.), Quantitative psychology research: The 80th annual meeting of the psychometric society (pp. 293-306). Springer. doi: https://doi.org/10.1007/978-3-319-19977-121

Kosorok, M. R., \& Moodie, E. E. M. (2015). Adaptive treatment strategies in practice (E. E. M. Moodie \& M. R. Kosorok, Eds.). Society for Industrial and Applied Mathematics. doi: 10.1137/1.9781611974188

Leite, W. L. (2016). Practical propensity score methods using R. Sage Publications. doi: https://doi.org/10.4135/9781071802854

Li, L., Lu, Y., \& Zhou, D. (2017). Provably optimal algorithms for generalized linear contextual bandits. In Proceedings of the 34th international conference on machine learning-volume 70 (pp. 2071-2080).

Lunceford, J. K., \& Davidian, M. (2004). Stratification and weighting via the propensity score in estimation of causal treatment effects: a comparative study. Statistics in Medicine, 23(19), 2937-2960. doi: https://doi.org/10.1002/sim.1903

Martin, M. O., Mullis, I. V. S., \& Hooper, M. (2016). Methods and procedures in timss 2015. TIMSS \& PIRLS International Study Center, Boston College. Retrieved from http://timssandpirls.bc.edu/publications/timss/2015-methods.html

McCaffrey, D. F., Ridgeway, G., \& Morral, A. R. (2004). Propensity score estimation with boosted regression for evaluating causal effects in observational studies. Psychological Methods, 9(4), 403. doi: https://doi.org/10.1037/1082-989X.9.4.403

Meyers, J. L., \& Beretvas, S. N. (2006). The impact of inappropriate modeling of crossclassified data structures. Multivariate Behavioral Research, 41(4), 473-497. doi: https://doi.org/10.1207/s15327906mbr41043

Neyman, J. S. (1923). On the application of probability theory to agricultural experiments: essay on principles. section 9 (with discussion). Statistical Science, 4, 465-480.

R Core Team. (2020). R: A language and environment for statistical computing [Computer software manual]. Vienna, Austria. Retrieved from https://www.R-project.org/

Raudenbush, S. W., \& Bryk, A. S. (2002). Hierarchical linear models: Applications and data analysis methods (Vol. 1). Sage. 
Rickles, J. H., \& Seltzer, M. (2014). A two-stage propensity score matching strategy for treatment effect estimation in a multisite observational study. Journal of Educational and Behavioral Statistics, 39(6), 612-636. doi: https://doi.org/10.3102/1076998614559748

Robins, J. M., Rotnitzky, A., \& Zhao, L. P. (1994). Estimation of regression coefficients when some regressors are not always observed. Journal of the American Statistical Association, 89 (427), 846-866. doi: https://doi.org/10.1080/01621459.1994.10476818

Rosenbaum, P. R., \& Rubin, D. B. (1983). The central role of the propensity score in observational studies for causal effects. Biometrika, 70, 41-55. doi: https://doi.org/10.1093/biomet/70.1.41

Rubin, D. B. (1974). Estimating causal effects of treatments in randomized and nonrandomized studies. Journal of Educational Psychology, 66(5), 688-701. doi: https://doi.org/10.1037/h0037350

Rubin, D. B. (1986). Comment: Which ifs have causal answers. Journal of the American Statistical Association, 81 (396), 961-962. doi: https://doi.org/10.1080/01621459.1986.10478355

Rubin, D. B. (2001). Using propensity scores to help design observational studies: application to the tobacco litigation. Health Services and Outcomes Research Methodology, 2(3-4), 169-188. doi: https://doi.org/10.1023/A:1020363010465

Schafer, J. L., \& Kang, J. (2008). Average causal effects from nonrandomized studies: a practical guide and simulated example. Psychological Methods, 13(4), 279. doi: https://doi.org/10.1037/a0014268

Scharfstein, D. O., Rotnitzky, A., \& Robins, J. M. (1999). Adjusting for nonignorable drop-out using semiparametric nonresponse models. Journal of the American Statistical Association, 94 (448), 1096-1120. doi: https://doi.org/10.1080/01621459.1999.10473862

Shadish, W. R., Clark, M. H., \& Steiner, P. M. (2008). Can nonrandomized experiments yield accurate answers? a randomized experiment comparing random and nonrandom assignments. Journal of the American Statistical Association, 103(484), 1334-1344. doi: https://doi.org/10.1198/016214508000000733

Steiner, P. M., Cook, T. D., Shadish, W. R., \& Clark, M. H. (2010). The importance of covariate selection in controlling for selection bias in observational studies. Psychological Methods, 15 (3), 250. doi: https://doi.org/10.1037/a0018719

Steiner, P. M., Kim, J.-S., \& Thoemmes, F. (2012). Matching strategies for observational multilevel data. Joint Statistical Meeting Proceedings, Social Statistics Section, 5020-5032.

Stuart, E. A. (2010). Matching methods for causal inference: A review and a look forward. Statistical Science, 25(1), 1. doi: https://doi.org/10.1214/09-STS313

Thoemmes, F. J., \& West, S. G. (2011). The use of propensity scores for nonrandomized designs with clustered data. Multivariate Behavioral Research, 46(3), 514-543. doi: https://doi.org/10.1080/00273171.2011.569395

Tibshirani, J., Athey, S., Friedberg, R., Hadad, V., Miner, L., Wager, S., \& Wright, M. (2019). grf: Generalized random forests (beta) [Computer software manual]. Retrieved from https://CRAN.R-project.org/package=grf (R package version 0.10.3)

van der Laan, M. J., Polley, E. C., \& Hubbard, A. E. (2007). Super learner. Statistical Applications in Genetics and Molecular Biology, 6(1). doi: https://doi.org/10.2202/1544-6115.1309

van der Laan, M. J., \& Rose, S. (2011). Targeted learning: causal inference for observational and experimental data. Springer Science \& Business Media.

Wager, S., \& Athey, S. (2018). Estimation and inference of heterogeneous treatment effects using random forests. Journal of the American Statistical Association, 113(523), 1228-1242. doi: https://doi.org/10.1080/01621459.2017.1319839

Westreich, D., Lessler, J., \& Funk, M. J. (2010). Propensity score estimation: neural networks, support vector machines, decision trees (cart), and meta-classifiers as alternatives to logistic regression. Journal of Clinical Epidemiology, 63(8), 826-833. doi: https://doi.org/10.1016/j.jclinepi.2009.11.020 
Wooldridge, J. M. (2010). Econometric analysis of cross section and panel data. MIT press. 


\section{A Data Generating Model for Three-level Data}

1. For each level-2 cluster $j=1, \ldots, J_{k}$ (e.g., classes) of level-3 cluster $k=1, \ldots, K$ (e.g., schools), generate number of individuals per cluster $n_{j k}$ by drawing a number from a normal distribution with mean $I$ and standard deviation $s d$ and rounding it to the nearest integer.

2. For each individual $i=1, \ldots, n_{j k}$ in level-2 cluster $j$ and level-3 cluster $k$, generate level-3, level-2, and level-1 covariates $\boldsymbol{Q}_{k}=\left(Q_{1 k}, Q_{2 k}\right), \boldsymbol{W}_{j k}=\left(W_{1 j k}, W_{2 j k}\right)$, and $\boldsymbol{X}_{i j k}=\left(X_{1 j k}, X_{2 j k}\right)$ as follows.

$$
\begin{aligned}
Q_{1 k} & \sim U[0,1], Q_{2 k} \sim U[0,1] \\
\left(\begin{array}{l}
W_{1 j k} \\
W_{2 j k}
\end{array}\right) & \sim N\left[\left(\begin{array}{c}
0.1 Q_{1 k}+0.05 Q_{2 k}+\kappa_{1 k} \\
0.08 Q_{1 k}+0.1 Q_{2 k}+\kappa_{2 k}
\end{array}\right), \quad\left(\begin{array}{cc}
2 & 0.2 \\
0.2 & 2
\end{array}\right)\right] \\
\left(\begin{array}{l}
X_{1 i j k} \\
X_{2 i j k}
\end{array}\right) & \sim N\left[\left(\begin{array}{c}
0.1 W_{1 j k}+0.05 W_{2 j k}+0.1 Q_{1 k}+0.02 Q_{2 k}+\kappa_{1 j k} \\
0.08 W_{1 j k}+0.1 W_{2 j k}+0.05 Q_{1 k}+0.01 Q_{2 k}+\kappa_{2 j k}
\end{array}\right), \quad\left(\begin{array}{cc}
10 & 2 \\
2 & 15
\end{array}\right)\right] \\
\left(\begin{array}{l}
\kappa_{1 k} \\
\kappa_{2 k}
\end{array}\right) & \sim N\left[\left(\begin{array}{l}
0 \\
0
\end{array}\right), \quad\left(\begin{array}{cc}
0.5 & 0 \\
0 & 0.5
\end{array}\right)\right],\left(\begin{array}{l}
\kappa_{1 j k} \\
\kappa_{2 j k}
\end{array}\right) \sim N\left[\left(\begin{array}{l}
0 \\
0
\end{array}\right), \quad\left(\begin{array}{cc}
1 & 0.1 \\
0.1 & 1
\end{array}\right)\right]
\end{aligned}
$$

Note that level-2 covariates form a hierarchical model with level-3 covariates $Q_{1 k}, Q_{2 k}$, random errors $\kappa_{1 k}, \kappa_{2 k}$, and random variances. Similarly, the means of level-1 covariates are a function of upper-level covariates $W_{1 j k}, W_{2 j k}, Q_{1 k}, Q_{2 k}$.

3. Generate individual treatment status $Z_{i j}$ from the following random-effects logistic selection model.

$$
\begin{aligned}
& \operatorname{logit}\left(\pi_{i j k}\right)=-0.2+0.1 X_{1 i j k}+0.03 X_{2 i j k}+0.1 W_{1 j k}+0.08 W_{2 j k}+0.1 Q_{1 k}+0.05 Q_{2 k}+R_{j k}^{W}+R_{k}^{Q} \\
& R_{j k}^{W} \sim N(0,1), R_{k}^{Q} \sim N(0,1) \\
& Z_{i j k} \sim \operatorname{Bernoulli}\left(\pi_{i j k}\right)
\end{aligned}
$$

where $\pi_{i j k}$ is the propensity score for individual $i$ in level-2 cluster $j$ and level-3 cluster $k$, and $R_{j k}^{W}$ and $R_{k}^{Q}$ are normally distributed random effects for level-2 clusters and level-3 clusters, respectively.

4. Generate the potential outcomes $Y_{i j k}(1), Y_{i j k}(0)$ and observed outcome $Y_{i j k}$ from random-effects linear regression models.

$$
\begin{aligned}
& Y_{i j k}(z)=100+\tau \cdot z+2 X_{1 i j k}+1 X_{2 i j k}+2 W_{1 j k}+1.5 W_{2 j k}+1 Q_{1 k}+0.5 Q_{2 k}+U_{j k}^{W}+U_{k}^{Q}+\epsilon_{i j k} \\
& \quad U_{j k}^{W} \sim N(0,10), U_{k}^{Q} \sim N(0,7), \epsilon_{i j k} \sim N(0,100) \\
& Y_{i j k}=Z_{i j k} Y_{i j k}(1)+\left(1-Z_{i j k}\right) Y_{i j k}(0)
\end{aligned}
$$

where $U_{j k}^{W}$ and $U_{k}^{Q}$ are normally distributed random effects for level-2 cluster $j$ of level-3 cluster $k$, respectively. $\epsilon_{i j k}$ is the random error for individual $i$ in level-2 cluster $j$ of level-3 cluster $k$. Also, the treatment effect is constant and set to $\tau=2$.

\section{B Data Generating Model for Cross-classified Data}

1. For each factor- 1 cluster $j=1, \ldots, J$, generate number of individuals (e.g., students) per cluster $n_{j}$ (e.g., schools) by drawing a number from a normal distribution with mean $n I$ and standard deviation $s d$ and rounding it to the nearest integer.

2. Create factor-2 Cluster labels $k=1, \ldots, K$, (e.g., neighborhoods) according to Meyers and Beretvas (2006). Here, suppose there is no correlation between residuals and there are three feeders from one factor to the other factor. Each factor- 1 cluster sends $70 \%$ of its individuals to the most adjacent factor- 2 cluster, $15 \%$ of its individuals to the next closest factor- 2 cluster, and $15 \%$ of its individuals to the third closest. The first factor- 1 cluster sends $80 \%$ of its individuals to the most adjacent factor- 2 cluster, and the rest of $20 \%$ to the next closest. Individuals of the last factor- 1 cluster are distributed in the same way as the first factor-1 cluster.

3. For each individual $i=1, \ldots, n_{(j k)}$ in factor-1 cluster $j$ and factor-2 cluster $k$, generate factor1 , factor-2, and individual-level covariates $\boldsymbol{W}_{j}=\left(W_{1 j}, W_{2 j}\right), \boldsymbol{Q}_{k}=\left(Q_{1 k}, Q_{2 k}\right)$ and $\boldsymbol{X}_{i(j k)}=$ 
$\left(X_{1 i(j k)}, X_{2 i(j k)}\right)$ as follows.

$$
\begin{aligned}
\left(\begin{array}{l}
W_{1 j} \\
W_{2 j}
\end{array}\right) & \sim N\left[\left(\begin{array}{l}
0 \\
0
\end{array}\right), \quad\left(\begin{array}{cc}
2 & 0.2 \\
0.2 & 2
\end{array}\right)\right] \\
\left(\begin{array}{l}
Q_{1 k} \\
Q_{2 k}
\end{array}\right) & \sim N\left[\left(\begin{array}{l}
0 \\
0
\end{array}\right), \quad\left(\begin{array}{cc}
1 & 0.1 \\
0.1 & 1
\end{array}\right)\right] \\
\left(\begin{array}{l}
X_{1 i(j k)} \\
X_{2 i(j k)}
\end{array}\right) & \sim N\left[\left(\begin{array}{l}
0.1 W_{1 j}+0.05 W_{2 j}+\kappa_{1 j} \\
0.08 W_{1 j}+0.1 W_{2 j}+\kappa_{2 j}
\end{array}\right), \quad\left(\begin{array}{cc}
10 & 2 \\
2 & 15
\end{array}\right)\right] \\
\left(\begin{array}{l}
\kappa_{1 j} \\
\kappa_{2 j}
\end{array}\right) & \sim N\left[\left(\begin{array}{l}
0 \\
0
\end{array}\right), \quad\left(\begin{array}{cc}
1 & 0.1 \\
0.1 & 1
\end{array}\right)\right]
\end{aligned}
$$

Note that individual-level covariates form a hierarchical model with factor-1 covariates $W_{1 j}, W_{2 j}$, random errors $\kappa_{1 j}, \kappa_{2 j}$, and random variances.

4. Generate individual treatment status $Z_{i(j k)}$ from the following cross-classified random-effects logistic propensity score model.

$$
\begin{aligned}
& \operatorname{logit}\left(\pi_{i j k}\right)=-0.2+0.1 X_{1 i(j k)}+0.03 X_{2 i(j k)}+0.1 W_{1 j}+0.08 W_{2 j}+0.1 Q_{1 k}+0.05 Q_{2 k}+R_{j}^{W}+R_{k}^{Q} \\
& R_{j}^{W} \sim N(0,1), \quad R_{k}^{Q} \sim N(0,0.5) \\
& Z_{i(j k)} \sim \operatorname{Bernoulli}\left(\pi_{i(j k)}\right)
\end{aligned}
$$

where $\pi_{i(j k)}$ is the propensity score for individual $i$ in cluster $j . R_{j}^{W}$ and $R_{k}^{Q}$ are normally distributed random effects for Factor-1 cluster $j$ and Factor-2 cluster $k$, respectively.

5. Generate the potential outcomes $Y_{i(j k)}(1), Y_{i(j k)}(0)$ and observed outcome $Y_{i(j k)}$ from cross-classified random-effects linear regression models.

$$
\begin{gathered}
Y_{i(j k)}(z)=100+\tau \cdot z+2 X_{1 i(j k)}+1 X_{2 i(j k)}+2 W_{1 j}+1.5 W_{2 j}+1 Q_{1 k}+0.5 Q_{2 k}+U_{j}^{W}+U_{k}^{Q}+\epsilon_{i(j k)} \\
\quad U_{j}^{W} \sim N(0,10), \quad U_{k}^{Q} \sim N(0,7), \quad \epsilon_{i j k} \sim N(0,100) \\
Y_{i(j k)}=Z_{i(j k)} Y_{i(j k)}(1)+\left(1-Z_{i(j k)}\right) Y_{i(j k)}(0)
\end{gathered}
$$

where $U_{j}^{W}$ and $U_{k}^{Q}$ are normally distributed random effects for factor- 1 cluster $j$ and factor-2 cluster $k$, respectively. $\epsilon_{i(j k)}$ is the random error for individual $i$ in factor- 1 cluster $j$ and factor- 2 cluster $k$. Also, the treatment effect is constant and set to $\tau=2$.

\section{Choosing the Number of Strata $S$ in MMW-S}

For each simulation condition, we chose the number of strata $S$ based on covariates' standardized mean differences and variance ratios (Hong, 2010). Specifically, we computed the mean number of balanced covariates across 200 replications and chose the optimal number based on within-stratum covariate balance (i.e., "mean.bal.s" and "var.bal.s" in tables below). Good balance of each covariate is achieved when the absolute standardized mean differences are smaller than 0.1 and variance ratios are in between $4 / 5$ and 5/4 (Rubin, 2001; Shadish et al., 2008; Steiner et al., 2010). Since we use 4 covariates in our two-level data simulation, the maximum number of balanced covariates is 4 . In both three-level data and cross-classified data, we use 6 covariates and thus, the maximum number of balanced covariates is 6 .

The tables below show how different measures of balance change as a function of $S$ along with the MSE of the MMW-S estimator. We saw that the choice of $S$ impacts the performance of MMW-S and in many cases, $S$ chosen based on Hong (2010) does not always lead to an estimator with the smallest MSE. 
Table 2: Covariate balance with varying numbers of strata in two-level data under Design 1: $\left(150,30_{(2)}\right)-3$ strata

\begin{tabular}{rcccccc}
\hline Num. strata & mean.bal.s & var.bal.s & lps.mean.dif.s & mean.bal.o & var.bal.o & MSE \\
\hline 1 & 0.615 & 3.920 & 0.952 & 0.615 & 3.920 & 8.125 \\
2 & 3.232 & 3.982 & 0.355 & 3.800 & 4.000 & 0.674 \\
3 & 3.595 & 3.947 & 0.202 & 4.000 & 4.000 & 0.214 \\
4 & 3.515 & 3.905 & 0.136 & 4.000 & 4.000 & 0.135 \\
5 & 3.365 & 3.856 & 0.101 & 4.000 & 4.000 & 0.141 \\
6 & 3.182 & 3.787 & 0.079 & 4.000 & 4.000 & 0.154 \\
7 & 3.037 & 3.694 & 0.064 & 4.000 & 4.000 & 0.171 \\
8 & 2.876 & 3.642 & 0.054 & 4.000 & 4.000 & 0.185 \\
9 & 2.737 & 3.536 & 0.047 & 4.000 & 4.000 & 0.200 \\
10 & 2.631 & 3.482 & 0.041 & 4.000 & 4.000 & 0.215 \\
11 & 2.538 & 3.414 & 0.036 & 4.000 & 4.000 & 0.223 \\
12 & 2.466 & 3.330 & 0.033 & 4.000 & 4.000 & 0.232 \\
13 & 2.373 & 3.263 & 0.030 & 4.000 & 4.000 & 0.243 \\
14 & 2.299 & 3.176 & 0.028 & 4.000 & 4.000 & 0.246 \\
15 & 2.248 & 3.115 & 0.026 & 4.000 & 4.000 & 0.253 \\
16 & 2.152 & 3.038 & 0.024 & 4.000 & 4.000 & 0.265 \\
17 & 2.120 & 2.990 & 0.023 & 4.000 & 4.000 & 0.263 \\
18 & 2.073 & 2.927 & 0.022 & 4.000 & 4.000 & 0.261 \\
19 & 1.998 & 2.893 & 0.020 & 4.000 & 4.000 & 0.273 \\
20 & 1.978 & 2.827 & 0.020 & 4.000 & 4.000 & 0.275 \\
\hline
\end{tabular}

Note: mean.bal.s and var.bal.s represent the number of covariates that is balanced in terms of standardized mean differences and variance ratios within each stratum, respectively. lps.mean.dif.s represents the mean absolute difference in the logit propensity score within each stratum. mean.bal.o and var.bal.o represent the number of covariates that is balanced in terms of standardized mean differences and variance ratios across strata, respectively. MSE represents the mean-squared error of the marginal mean weighting through stratification (MMW-S) estimator. 
Table 3: Covariate balance with varying numbers of strata in two-level data under Design 1: $\left(150,30_{(15)}\right)-4$ strata

\begin{tabular}{rcccccc}
\hline Num. strata & mean.bal.s & var.bal.s & lps.mean.dif.s & mean.bal.o & var.bal.o & MSE \\
\hline 1 & 0.600 & 3.895 & 0.955 & 0.600 & 3.895 & 8.061 \\
2 & 3.150 & 3.970 & 0.355 & 3.645 & 4.000 & 0.719 \\
3 & 3.512 & 3.935 & 0.201 & 3.995 & 4.000 & 0.217 \\
4 & 3.534 & 3.901 & 0.136 & 4.000 & 4.000 & 0.147 \\
5 & 3.364 & 3.856 & 0.101 & 4.000 & 4.000 & 0.131 \\
6 & 3.190 & 3.796 & 0.078 & 4.000 & 4.000 & 0.138 \\
7 & 3.039 & 3.705 & 0.063 & 4.000 & 4.000 & 0.151 \\
8 & 2.863 & 3.639 & 0.053 & 4.000 & 4.000 & 0.157 \\
9 & 2.761 & 3.554 & 0.045 & 4.000 & 4.000 & 0.165 \\
10 & 2.667 & 3.470 & 0.040 & 4.000 & 4.000 & 0.175 \\
11 & 2.573 & 3.413 & 0.036 & 4.000 & 4.000 & 0.183 \\
12 & 2.468 & 3.336 & 0.032 & 4.000 & 4.000 & 0.190 \\
13 & 2.400 & 3.275 & 0.029 & 4.000 & 4.000 & 0.194 \\
14 & 2.324 & 3.199 & 0.027 & 4.000 & 4.000 & 0.201 \\
15 & 2.233 & 3.121 & 0.025 & 3.995 & 3.995 & 0.204 \\
16 & 2.221 & 3.083 & 0.023 & 4.000 & 3.995 & 0.208 \\
17 & 2.140 & 2.996 & 0.022 & 3.995 & 3.995 & 0.210 \\
18 & 2.092 & 2.967 & 0.021 & 3.995 & 3.995 & 0.206 \\
19 & 2.056 & 2.893 & 0.019 & 4.000 & 3.995 & 0.208 \\
20 & 1.987 & 2.845 & 0.019 & 4.000 & 3.995 & 0.214 \\
\hline
\end{tabular}

Note: mean.bal.s and var.bal.s represent the number of covariates that is balanced in terms of standardized mean differences and variance ratios within each stratum, respectively. lps.mean.dif.s represents the mean absolute difference in the logit propensity score within each stratum. mean.bal.o and var.bal.o represent the number of covariates that is balanced in terms of standardized mean differences and variance ratios across strata, respectively. MSE represents the mean-squared error of the marginal mean weighting through stratification (MMW-S) estimator. 
Table 4: Covariate balance with varying numbers of strata in two-level data under Design 1: $\left(30,150_{(4)}\right)-5$ strata

\begin{tabular}{rcccccc}
\hline Num. strata & mean.bal.s & var.bal.s & lps.mean.dif.s & mean.bal.o & var.bal.o & MSE \\
\hline 1 & 0.775 & 3.425 & 0.953 & 0.775 & 3.425 & 7.617 \\
2 & 2.445 & 3.703 & 0.354 & 2.835 & 3.860 & 0.940 \\
3 & 3.013 & 3.787 & 0.200 & 3.810 & 3.965 & 0.392 \\
4 & 3.189 & 3.777 & 0.134 & 3.965 & 3.985 & 0.242 \\
5 & 3.216 & 3.750 & 0.099 & 3.985 & 3.995 & 0.191 \\
6 & 3.114 & 3.712 & 0.076 & 3.995 & 4.000 & 0.167 \\
7 & 3.026 & 3.676 & 0.062 & 4.000 & 4.000 & 0.156 \\
8 & 2.908 & 3.607 & 0.052 & 4.000 & 4.000 & 0.148 \\
9 & 2.792 & 3.544 & 0.043 & 4.000 & 4.000 & 0.145 \\
10 & 2.691 & 3.470 & 0.038 & 4.000 & 4.000 & 0.153 \\
11 & 2.623 & 3.415 & 0.034 & 4.000 & 4.000 & 0.146 \\
12 & 2.526 & 3.354 & 0.030 & 4.000 & 4.000 & 0.147 \\
13 & 2.423 & 3.311 & 0.027 & 4.000 & 4.000 & 0.146 \\
14 & 2.373 & 3.243 & 0.025 & 4.000 & 4.000 & 0.146 \\
15 & 2.300 & 3.181 & 0.023 & 4.000 & 4.000 & 0.142 \\
16 & 2.227 & 3.140 & 0.021 & 4.000 & 4.000 & 0.142 \\
17 & 2.194 & 3.084 & 0.020 & 4.000 & 4.000 & 0.144 \\
18 & 2.146 & 3.031 & 0.018 & 4.000 & 4.000 & 0.142 \\
19 & 2.086 & 2.972 & 0.018 & 4.000 & 4.000 & 0.142 \\
20 & 2.026 & 2.932 & 0.016 & 4.000 & 4.000 & 0.143 \\
\hline
\end{tabular}

Note: mean.bal.s and var.bal.s represent the number of covariates that is balanced in terms of standardized mean differences and variance ratios within each stratum, respectively. lps.mean.dif.s represents the mean absolute difference in the logit propensity score within each stratum. mean.bal.o and var.bal.o represent the number of covariates that is balanced in terms of standardized mean differences and variance ratios across strata, respectively. MSE represents the mean-squared error of the marginal mean weighting through stratification (MMW-S) estimator. 
Table 5: Covariate balance with varying numbers of strata in two-level data under Design 1: $\left(5000,10_{(0.5)}\right)-2$ strata

\begin{tabular}{rcccccc}
\hline Num. strata & mean.bal.s & var.bal.s & lps.mean.dif.s & mean.bal.o & var.bal.o & MSE \\
\hline 1 & 0.070 & 4.000 & 0.922 & 0.070 & 4 & 8.823 \\
2 & 4.000 & 4.000 & 0.346 & 4.000 & 4 & 0.113 \\
3 & 3.935 & 4.000 & 0.198 & 4.000 & 4 & 0.108 \\
4 & 3.380 & 4.000 & 0.134 & 3.935 & 4 & 0.364 \\
5 & 2.897 & 4.000 & 0.100 & 3.005 & 4 & 0.574 \\
6 & 2.666 & 3.994 & 0.078 & 3.000 & 4 & 0.730 \\
7 & 2.493 & 3.982 & 0.063 & 2.980 & 4 & 0.836 \\
8 & 2.352 & 3.969 & 0.053 & 2.940 & 4 & 0.910 \\
9 & 2.259 & 3.953 & 0.045 & 2.845 & 4 & 0.968 \\
10 & 2.188 & 3.939 & 0.039 & 2.745 & 4 & 1.011 \\
11 & 2.126 & 3.929 & 0.035 & 2.635 & 4 & 1.039 \\
12 & 2.095 & 3.912 & 0.031 & 2.515 & 4 & 1.065 \\
13 & 2.044 & 3.903 & 0.028 & 2.425 & 4 & 1.077 \\
14 & 2.024 & 3.894 & 0.025 & 2.320 & 4 & 1.093 \\
15 & 2.005 & 3.883 & 0.023 & 2.225 & 4 & 1.101 \\
16 & 1.991 & 3.875 & 0.021 & 2.195 & 4 & 1.110 \\
17 & 1.963 & 3.861 & 0.019 & 2.135 & 4 & 1.116 \\
18 & 1.934 & 3.853 & 0.018 & 2.085 & 4 & 1.121 \\
19 & 1.948 & 3.843 & 0.017 & 2.000 & 4 & 1.122 \\
20 & 1.925 & 3.836 & 0.016 & 1.950 & 4 & 1.124 \\
\hline
\end{tabular}

Note: mean.bal.s and var.bal.s represent the number of covariates that is balanced in terms of standardized mean differences and variance ratios within each stratum, respectively. lps.mean.dif.s represents the mean absolute difference in the logit propensity score within each stratum. mean.bal.o and var.bal.o represent the number of covariates that is balanced in terms of standardized mean differences and variance ratios across strata, respectively. MSE represents the mean-squared error of the marginal mean weighting through stratification (MMW-S) estimator. 
Table 6: Covariate balance with varying numbers of strata in two-level data under Design 1: $\left(10,5000_{(4)}\right)-15$ strata

\begin{tabular}{rcccccc}
\hline Num. strata & mean.bal.s & var.bal.s & lps.mean.dif.s & mean.bal.o & var.bal.o & MSE \\
\hline 1 & 0.720 & 2.965 & 0.947 & 0.720 & 2.965 & 7.292 \\
2 & 2.005 & 3.397 & 0.344 & 2.500 & 3.670 & 1.017 \\
3 & 2.622 & 3.623 & 0.186 & 3.350 & 3.885 & 0.307 \\
4 & 2.976 & 3.745 & 0.119 & 3.715 & 3.945 & 0.161 \\
5 & 3.215 & 3.839 & 0.083 & 3.845 & 3.965 & 0.133 \\
6 & 3.378 & 3.873 & 0.062 & 3.925 & 3.985 & 0.071 \\
7 & 3.496 & 3.856 & 0.048 & 3.980 & 3.990 & 0.053 \\
8 & 3.567 & 3.829 & 0.038 & 3.995 & 3.995 & 0.045 \\
9 & 3.638 & 3.833 & 0.029 & 4.000 & 4.000 & 0.049 \\
10 & 3.731 & 3.872 & 0.021 & 4.000 & 4.000 & 0.073 \\
11 & 3.738 & 3.861 & 0.019 & 4.000 & 4.000 & 0.039 \\
12 & 3.746 & 3.868 & 0.018 & 4.000 & 4.000 & 0.027 \\
13 & 3.767 & 3.879 & 0.016 & 4.000 & 4.000 & 0.023 \\
14 & 3.774 & 3.884 & 0.014 & 4.000 & 4.000 & 0.021 \\
15 & 3.791 & 3.892 & 0.013 & 4.000 & 4.000 & 0.023 \\
16 & 3.795 & 3.879 & 0.012 & 4.000 & 4.000 & 0.021 \\
17 & 3.787 & 3.868 & 0.011 & 4.000 & 4.000 & 0.020 \\
18 & 3.799 & 3.868 & 0.010 & 4.000 & 4.000 & 0.018 \\
19 & 3.804 & 3.863 & 0.008 & 4.000 & 4.000 & 0.017 \\
20 & 3.800 & 3.859 & 0.007 & 4.000 & 4.000 & 0.020 \\
\hline
\end{tabular}

Note: mean.bal.s and var.bal.s represent the number of covariates that is balanced in terms of standardized mean differences and variance ratios within each stratum, respectively. lps.mean.dif.s represents the mean absolute difference in the logit propensity score within each stratum. mean.bal.o and var.bal.o represent the number of covariates that is balanced in terms of standardized mean differences and variance ratios across strata, respectively. MSE represents the mean-squared error of the marginal mean weighting through stratification (MMW-S) estimator. 


\section{Simulation Results using Doubly Robust Estimators in Causal Forests}

The simulation results using the built-in function, average_treatment_effect, particularly applying a modified version of augmented inverse-propensity weighting (Robins, Rotnitzky, \& Zhao, 1994), in grf are provided below. We found that the mean estimator of the pointwise predictions consistently provided better estimates in terms of bias and efficiency than using the built-in average_treatment_effect function across simulation conditions.

Table 7: Performance of ATE estimates in two-level data under Design 1: using the built-in function average_treatment_effect in grf

\begin{tabular}{lrcrrrrrrr}
\hline$\left(\mathrm{J}_{(} \mathrm{I}_{(s d)}\right)$ & \multicolumn{3}{c}{$\left(150,30_{(2)}\right)$} & \multicolumn{4}{c}{$\left(150,30_{(15)}\right)$} & \multicolumn{3}{c}{$\left(30,150_{(4)}\right)$} \\
Estimator & $\operatorname{Bias}(\%)$ & $\mathrm{SD}$ & $\mathrm{MSE}$ & $\operatorname{Bias}(\%)$ & $\mathrm{SD}$ & $\mathrm{MSE}$ & $\operatorname{Bias}(\%)$ & \multicolumn{1}{c}{$\mathrm{SD}$} & $\mathrm{MSE}$ \\
\hline $\mathrm{CF}$ & 5.90 & 0.376 & 0.155 & 6.65 & 0.367 & 0.152 & 5.30 & 0.359 & 0.140 \\
$\mathrm{CF}+$ Est.PS & 4.20 & 0.362 & 0.138 & 4.75 & 0.354 & 0.134 & 1.75 & 0.351 & 0.124 \\
$\mathrm{CF}+\mathrm{ID}$ & 6.35 & 0.410 & 0.184 & 6.90 & 0.467 & 0.237 & 22.25 & 0.686 & 0.668 \\
$\mathrm{CF}+$ Est.PS+ID & 4.85 & 0.359 & 0.138 & 6.35 & 0.418 & 0.191 & 2.35 & 0.352 & 0.126 \\
\hline
\end{tabular}

Note: $J$ and $\mathrm{I}_{(s d)}$ represent the number of clusters and the mean size of clusters and its standard deviation, respectively. CF represents Causal Forests without modification. CF+Est.PS represents CF with propensity scores from a multilevel logistic regression. $\mathrm{CF}+\mathrm{ID}$ represents $\mathrm{CF}$ with cluster labels. $\mathrm{CF}+$ Est.PS $+\mathrm{ID}$ represents $\mathrm{CF}$ with propensity scores from a multilevel logistic regression and cluster labels.

Table 8: Performance of ATE estimates in two-level data: asymptotic properties using the built-in function average_treatment_effect in grf

\begin{tabular}{lrcrrrr}
\hline$\left(\mathrm{J}, \mathrm{I}_{(s d)}\right)$ & \multicolumn{3}{c}{$\left(5000,10_{(0.5)}\right)$} & \multicolumn{3}{c}{$\left(10,5000_{(4)}\right)$} \\
Estimator & $\operatorname{Bias}(\%)$ & $\mathrm{SD}$ & $\mathrm{MSE}$ & $\operatorname{Bias}(\%)$ & \multicolumn{1}{c}{$\mathrm{SD}$} & $\mathrm{MSE}$ \\
\hline $\mathrm{CF}$ & 1.45 & 0.104 & 0.012 & 0.85 & 0.107 & 0.012 \\
$\mathrm{CF}+$ Est.PS & 9.40 & 0.108 & 0.047 & 0.20 & 0.107 & 0.012 \\
$\mathrm{CF}+\mathrm{ID}$ & 1.30 & 0.104 & 0.011 & 43.00 & 1.375 & 2.628 \\
$\mathrm{CF}+$ Est.PS+ID & 9.50 & 0.108 & 0.048 & 0.25 & 0.109 & 0.012 \\
\hline
\end{tabular}

Note: $J$ and $\mathrm{I}_{(s d)}$ represent the number of clusters and the mean size of clusters and its standard deviation, respectively. CF represents Causal Forests without modification. CF+Est.PS represents $\mathrm{CF}$ with propensity scores from a multilevel logistic regression. $\mathrm{CF}+\mathrm{ID}$ represents $\mathrm{CF}$ with cluster labels. $\mathrm{CF}+$ Est.PS $+\mathrm{ID}$ represents $\mathrm{CF}$ with propensity scores from a multilevel logistic regression and cluster labels. 
Table 9: Performance of ATE estimates in three-level data: using the built-in function average_treatment_effect in grf

\begin{tabular}{lrrrrrrrrr}
\hline$\left(\mathrm{K}, \mathrm{J}_{(s d)}, \mathrm{I}_{(s d)}\right)$ & \multicolumn{4}{c}{$\left(100,15_{(1)}, 30_{(2)}\right)$} & \multicolumn{3}{c}{$\left(100,15_{(1)}, 30_{(2)}\right)$} & \multicolumn{3}{c}{$\left(30,15_{(1)}, 100_{(4)}\right)$} \\
Estimator & Bias(\%) & \multicolumn{1}{c}{$\mathrm{SD}$} & $\operatorname{MSE}$ & $\operatorname{Bias}(\%)$ & \multicolumn{1}{c}{$\mathrm{SD}$} & $\mathrm{MSE}$ & $\operatorname{Bias}(\%)$ & \multicolumn{1}{c}{$\mathrm{SD}$} & $\mathrm{MSE}$ \\
\hline $\mathrm{CF}$ & 8.20 & 0.148 & 0.049 & 4.45 & 0.138 & 0.027 & 4.75 & 0.140 & 0.028 \\
$\mathrm{CF}+$ Est.PS & 5.90 & 0.126 & 0.030 & 5.00 & 0.137 & 0.029 & 2.25 & 0.128 & 0.018 \\
$\mathrm{CF}+\mathrm{ID} 2$ & 6.50 & 0.194 & 0.055 & 3.55 & 0.152 & 0.028 & 8.50 & 0.277 & 0.106 \\
$\mathrm{CF}+\mathrm{ID} 3$ & 9.75 & 0.244 & 0.097 & 17.60 & 0.618 & 0.506 & 15.45 & 0.451 & 0.299 \\
$\mathrm{CF}+$ Est.PS+ID2 & 6.30 & 0.126 & 0.032 & 5.35 & 0.138 & 0.030 & 2.80 & 0.128 & 0.020 \\
$\mathrm{CF}+$ Est.PS+ID3 & 7.15 & 0.127 & 0.036 & 7.70 & 0.146 & 0.045 & 3.35 & 0.130 & 0.021 \\
\hline
\end{tabular}

Note: $K, \mathrm{~J}_{(s d)}$, and $\mathrm{I}_{(s d)}$ represent the number of level-3 clusters, the mean size of level-3 clusters with its standard deviation, and the mean size of level-2 clusters with its standard deviation, respectively. CF represents Causal Forests without modification. CF +Est.PS represents CF with propensity scores from a multilevel logistic regression. $\mathrm{CF}+\mathrm{ID} 2$ and $\mathrm{CF}+\mathrm{ID} 3$ represent $\mathrm{CF}$ with level-2 cluster labels and level-3 cluster labels, respectively. $\mathrm{CF}+$ Est.PS+ID2 and CF+Est.PS+ID3 represent CF with propensity scores from a multilevel logistic regression and level-2 or level-3 cluster labels.

Table 10: Performance of ATE estimates in cross-classified data: using the built-in function average_treatment_effect in grf

\begin{tabular}{|c|c|c|c|c|c|c|c|c|c|}
\hline$\left(\mathrm{F} 1, \mathrm{~F} 2, \mathrm{I}_{(s d)}\right)$ & \multicolumn{3}{|c|}{$\left(150,150,30_{(2)}\right)$} & \multicolumn{3}{|c|}{$\left(150,150,30_{(15)}\right)$} & \multicolumn{3}{|c|}{$\left(30,30,150_{(4)}\right)$} \\
\hline Estimator & $\operatorname{Bias}(\%)$ & SD & MSE & $\operatorname{Bias}(\%)$ & SD & MSE & $\operatorname{Bias}(\%)$ & SD & MSE \\
\hline $\mathrm{CF}$ & 11.45 & 0.400 & 0.212 & 10.80 & 0.397 & 0.204 & 6.20 & 0.429 & 0.199 \\
\hline $\mathrm{CF}+$ Est.PS & 9.05 & 0.389 & 0.184 & 7.85 & 0.389 & 0.176 & 1.30 & 0.392 & 0.154 \\
\hline $\mathrm{CF}+\mathrm{F} 1 \mathrm{ID}$ & 12.20 & 0.456 & 0.267 & 11.85 & 0.518 & 0.325 & 23.65 & 0.831 & 0.913 \\
\hline $\mathrm{CF}+\mathrm{F} 2 \mathrm{ID}$ & 11.00 & 0.448 & 0.249 & 10.70 & 0.469 & 0.266 & 18.55 & 0.764 & 0.721 \\
\hline $\mathrm{CF}+\mathrm{F} 12 \mathrm{ID}$ & 11.90 & 0.493 & 0.299 & 9.70 & 0.566 & 0.358 & 15.35 & 0.720 & 0.612 \\
\hline $\mathrm{CF}+$ Est.PS $+\mathrm{F} 1 \mathrm{ID}$ & 9.85 & 0.391 & 0.192 & 9.80 & 0.458 & 0.248 & 2.40 & 0.397 & 0.160 \\
\hline $\mathrm{CF}+$ Est.PS+F2ID & 9.60 & 0.391 & 0.190 & 8.95 & 0.416 & 0.205 & 2.20 & 0.397 & 0.159 \\
\hline $\mathrm{CF}+$ Est.PS $+\mathrm{F} 12 \mathrm{ID}$ & 11.90 & 0.484 & 0.291 & 10.25 & 0.557 & 0.352 & 3.35 & 0.512 & 0.266 \\
\hline
\end{tabular}

Note: F1, F2, and $\mathrm{I}_{(s d)}$ represent the number of factor-1 clusters, the number of factor-2 clusters, and the mean size of factor-1 clusters with its standard deviation, respectively. CF represents Causal Forests without modification. $\mathrm{CF}+$ Est.PS represents $\mathrm{CF}$ with propensity scores from a multilevel logistic regression. $\mathrm{CF}+\mathrm{F} 1 \mathrm{ID}, \mathrm{CF}+\mathrm{F} 2 \mathrm{ID}$, and $\mathrm{CF}+\mathrm{F} 12 \mathrm{ID}$ represent $\mathrm{CF}$ with the first factor labels, the second factor labels, and the two factors' combined labels, respectively. $\mathrm{CF}+$ Est.PS + F1ID, $\mathrm{CF}+$ Est.PS + F2ID, and $\mathrm{CF}+$ Est.PS + F12ID represent CF with propensity scores from a multilevel logistic regression and the first factor, the second factor, or the combined labels. 
Table 11: Performance of ATE estimates in two-level data under Design 2: using the built-in function average_treatment_effect in grf

\begin{tabular}{|c|c|c|c|c|c|c|c|c|c|}
\hline \multirow{2}{*}{$\begin{array}{l}\left(\mathrm{J}, \mathrm{I}_{(s d)}\right) \\
\text { Estimator }\end{array}$} & \multicolumn{3}{|c|}{$\beta_{1}=0.02, \beta_{2}=0.3$} & \multicolumn{3}{|c|}{$\beta_{1}=0.04, \beta_{2}=0.6$} & \multicolumn{3}{|c|}{$\beta_{1}=0.06, \beta_{2}=1$} \\
\hline & $\operatorname{Bias}(\%)$ & SD & MSE & $\operatorname{Bias}(\%)$ & $\mathrm{SD}$ & MSE & $\operatorname{Bias}(\%)$ & $\mathrm{SD}$ & MSE \\
\hline \multicolumn{10}{|l|}{$\left(150,30_{(2)}\right)$} \\
\hline $\mathrm{CF}$ & 7.05 & 0.385 & 0.168 & 7.40 & 0.387 & 0.172 & 11.80 & 0.382 & 0.202 \\
\hline $\mathrm{CF}+$ Est.PS & 6.20 & 0.372 & 0.154 & 9.80 & 0.376 & 0.180 & 16.55 & 0.368 & 0.245 \\
\hline $\mathrm{CF}+\mathrm{ID}$ & 7.90 & 0.419 & 0.201 & 9.95 & 0.425 & 0.220 & 15.75 & 0.420 & 0.276 \\
\hline $\mathrm{CF}+$ Est.PS $+\mathrm{ID}$ & 7.50 & 0.370 & 0.160 & 13.65 & 0.374 & 0.214 & 22.55 & 0.369 & 0.340 \\
\hline \multicolumn{10}{|l|}{$\left(150,30_{(15)}\right)$} \\
\hline $\mathrm{CF}$ & 6.95 & 0.449 & 0.221 & 7.30 & 0.450 & 0.223 & 11.70 & 0.448 & 0.256 \\
\hline $\mathrm{CF}+$ Est.PS & 7.45 & 0.415 & 0.194 & 10.8 & 0.419 & 0.223 & 17.85 & 0.414 & 0.299 \\
\hline $\mathrm{CF}+\mathrm{ID}$ & 8.30 & 0.477 & 0.255 & 10.45 & 0.480 & 0.274 & 16.30 & 0.478 & 0.334 \\
\hline $\mathrm{CF}+$ Est.PS & 8.65 & 0.415 & 0.202 & 14.90 & 0.421 & 0.266 & 24.35 & 0.414 & 0.408 \\
\hline \multicolumn{10}{|l|}{$\left(30,150_{(4)}\right)$} \\
\hline $\mathrm{CF}$ & 5.40 & 0.380 & 0.156 & 4 . & 0.398 & 0.168 & 8.60 & 0.383 & 0.176 \\
\hline $\mathrm{CF}+$ Est.PS & 2.65 & 0.373 & 0.141 & 5.00 & 0.393 & 0.164 & 10.65 & 0.377 & 0.187 \\
\hline $\mathrm{CF}+\mathrm{ID}$ & 25.00 & 0.707 & 0.750 & 33.60 & 0.731 & 0.987 & 46.00 & 0.734 & 1.384 \\
\hline $\mathrm{CF}+$ Est.PS $+\mathrm{ID}$ & 5.95 & 0.375 & 0.155 & 19.95 & 0.414 & 0.331 & 38.00 & 0.433 & 0.765 \\
\hline
\end{tabular}

Note: $J$ and $\mathrm{I}_{(s d)}$ represent the number of clusters and the mean size of clusters and its standard deviation, respectively. CF represents Causal Forests without modification. CF + Est.PS represents CF with propensity scores from a multilevel logistic regression. $\mathrm{CF}+\mathrm{ID}$ represents $\mathrm{CF}$ with cluster labels. $\mathrm{CF}+$ Est.PS $+\mathrm{ID}$ represents $\mathrm{CF}$ with propensity scores from a multilevel logistic regression and cluster labels. 